\title{
NON-VANISHING OF THE SYMMETRIC SQUARE $L$-FUNCTION AT THE CENTRAL POINT
}

\author{
RIZWANUR KHAN
}

\begin{abstract}
Using the mollifier method, we show that for a positive proportion of holomorphic Hecke eigenforms of level one and weight bounded by a large enough constant, the associated symmetric square $L$-function does not vanish at the central point of its critical strip. We note that our proportion is the same as that found by other authors for other families of $L$-functions also having symplectic symmetry type.
\end{abstract}

\section{INTRODUCTION}

A recurring theme in the theory of $L$-functions is the study of whether or not an $L$-function vanishes at its central point (the point of symmetry of the $L$-function's functional equation). One may be interested in this question if the central value is expected to be a special value, as in the case of the $L$-function associated to an elliptic curve over a number field, for which the Birch and Swinnerton-Dyer Conjecture predicts that the order of vanishing at the central point is the same as the rank of the elliptic curve. Even if the central value of the $L$-function under study is not expected to be a special value, the question of non-vanishing is connected to deep conjectures on the distribution of the $L$-function's zeros. The following type of problem is usually formulated: given a suitable collection of related $L$ functions ordered by analytic conductor, how much of this family is non-vanishing at the central point? A great deal of work has been done on this problem for many families of $L$-functions, of which only a small selection will be referenced in the course of this paper. Here we study the problem for a family of degree three $L$-functions: symmetric square $L$-functions lifted from classical cusp forms.

Let $H_{k}$ denote the set of holomorphic cusp forms $f$ of level one and weight $k$ which are eigenfunctions of every Hecke operator and have Fourier expansions of the form

$$
f(z)=\sum_{n=1}^{\infty} a_{f}(n) n^{\frac{k-1}{2}} e^{2 \pi i n z}
$$

for $\Im(z)>0$ with $a_{f}(1)=1$. The coefficients $a_{f}(n)$ are necessarily real and satisfy the Ramanujan-Petersson bound $\left|a_{f}(n)\right| \leq d(n)$, where $d(n)$ is the divisor function. The set $H_{k}$ has $\frac{k}{12}+O(1)$ elements and forms an orthogonal basis of the space of cusp forms of level one and weight $k$ (see [6]). For $\Re(s)>1$ we can define the symmetric square $L$-function associated to $f \in H_{k}$ as the absolutely convergent

2000 Mathematics Subject Classification: 11M99. 
Dirichlet series

$$
L\left(s, \operatorname{sym}^{2} f\right)=\zeta(2 s) \sum_{n=1}^{\infty} \frac{a_{f}\left(n^{2}\right)}{n^{s}} .
$$

In terms of the Rankin-Selberg $L$-function of $f$, we have $L\left(s, \operatorname{sym}^{2} f\right)=\frac{\zeta(2 s) L(s, f \otimes f)}{\zeta(s)}$. The symmetric square $L$-function has the degree three Euler product

$$
L\left(s, \operatorname{sym}^{2} f\right)=\prod_{p}\left(1-a_{f}\left(p^{2}\right) p^{-s}+a_{f}\left(p^{2}\right) p^{-2 s}-p^{-3 s}\right)^{-1} .
$$

Shimura [16] showed that $L\left(s, \operatorname{sym}^{2} f\right)$ analytically extends to an entire function on $\mathbb{C}$ and satisfies the functional equation

$$
L_{\infty}(s) L\left(s, \operatorname{sym}^{2} f\right)=L_{\infty}(1-s) L\left(1-s, \operatorname{sym}^{2} f\right),
$$

where

$$
L_{\infty}(s)=\pi^{-\frac{3}{2} s} \Gamma\left(\frac{s+1}{2}\right) \Gamma\left(\frac{s+k-1}{2}\right) \Gamma\left(\frac{s+k}{2}\right)=\pi^{-\frac{3}{2} s+\frac{1}{2}} 2^{2-s-k} \Gamma\left(\frac{s+1}{2}\right) \Gamma(s+k-1) .
$$

Thus under our normalization the central point is $s=\frac{1}{2}$. We also see from the functional equation that the conductor of $L\left(s, \operatorname{sym}^{2} f\right)$ in the weight aspect is $k^{2}$. Gelbart and Jacquet 4 showed that $L\left(s, \operatorname{sym}^{2} f\right)$ is actually the $L$-function attached to a cusp form on $G L(3)$.

There is no trivial reason for $L\left(s, \operatorname{sym}^{2} f\right)$ to vanish at $s=\frac{1}{2}$; that is, the functional equation does not force $L\left(\frac{1}{2}, \operatorname{sym}^{2} f\right)$ to equal zero. There seems to be no non-trivial reason either, as $L\left(\frac{1}{2}, \operatorname{sym}^{2} f\right)$ is not known to be a special value. By this general principle and by the Density Conjecture, which we discuss in the next section, it is conjectured that $L\left(\frac{1}{2}, \operatorname{sym}^{2} f\right)$ is never zero. Indeed it would be a great achievement to prove that for a positive percentage of the family $H_{k}$ we have $L\left(\frac{1}{2}, \operatorname{sym}^{2} f\right) \neq 0$. We do not solve this problem here but settle for something weaker. We show a positive percentage of non-vanishing on a larger family: essentially $\bigcup_{\substack{K \leq k \leq 2 K \\ k \equiv 0 \\ \bmod 2}} H_{k}$ for large $K$. We are in the standard situation where the ratio of $\log$ (conductor) to $\log$ (family size) is 1 , while this ratio is 2 in the case of a family of an individual weight. In a different direction, Blomer [1 has considered symmetric square lifts of cusp forms $f$ of prime level $N$, real primitive nebentypus and fixed weight (so that the ratio $\log$ (conductor): $\log ($ family) is 1 ). He showed that for large $N$, we have $L\left(\frac{1}{2}, \operatorname{sym}^{2} f\right) \neq 0$ for a positive proportion of this family. However he did not compute an explicit proportion.

For any complex numbers $\alpha_{f}$ depending on $f$, let

$$
\sum_{f \in H_{k}}^{h} \alpha_{f}=\frac{2 \pi^{2}}{k-1} \sum_{f \in H_{k}} \frac{\alpha_{f}}{L\left(1, \operatorname{sym}^{2} f\right)} .
$$

denote the 'harmonic' average of $\alpha_{f}$. The weights $L\left(1, \mathrm{sym}^{2} f\right)^{-1}$ arise naturally from the Petersson trace formula. Note that $\sum_{f \in H_{k}}^{h} 1=1+O\left(2^{-k}\right)$ by the trace formula. Our main theorem is

Theorem 1.1. Let $h$ be a positive valued, infinitely differentiable and compactly supported function on the positive reals. We have for any $0<a<\frac{1}{2}$ fixed and large 
enough $K$,

$$
\sum_{k \equiv 0 \bmod 2} h\left(\frac{k}{K}\right) \sum_{\substack{f \in H_{k} \\ L\left(\frac{1}{2}, s y m^{2} f\right) \neq 0}}^{h} 1 \geq\left(1-\frac{1}{(1+a)^{3}}\right) \sum_{k \equiv 0 \bmod 2} h\left(\frac{k}{K}\right) \sum_{f \in H_{k}}^{h} 1 .
$$

For the rest of the paper, let us fix a function $h$ satisfying the conditions of the theorem. Thus taking $a$ arbitrarily close to $\frac{1}{2}$ and counting harmonically, we have that at least $\frac{19}{27}>70 \%$ of our family is non-vanishing at the central point. The reason for the theorem's presentation of the constant of proportionality in terms of a parameter $a$ rather than just the best numerical value will be explained.

We expect the same theorem to hold if we replace the sums $\sum^{h}$ by uniform sums $\frac{1}{H_{k} \mid} \sum$. We have only worked with harmonic sums here, but it would be interesting to remove the weights $L\left(1, \operatorname{sym}^{2} f\right)^{-1}$. A method to do this was described in [10. With less work, one can get a smaller but explicit uniform proportion of nonvanishing from our result using just the Cauchy-Schwarz inequality and moments of $L\left(1, \operatorname{sym}^{2} f\right)$.

1.2. The Katz-Sarnak Philosophy. In this section, we discuss a few instances relevant to our paper of the so-called Katz-Sarnak philosophy. Since the discovery of a connection between zeros of the Riemann Zeta function and eigenvalues of random matrices by Montgomery [13] and Dyson [3] in the 1970's, there has been a lot of work by many authors studying the distribution of zeros of $L$-functions in families. The conjectures stemming from this work (and the proofs in function field settings by Katz and Sarnak [9]) suggest that the distribution of zeros near the central point of a family of $L$-functions is the same as the distribution of eigenvalues with small argument of a corresponding classical compact group. Here we are concerned with the group $U S p(2 N)$ of $2 N \times 2 N$ complex matrices which are both unitary and symplectic.

In [7, Iwaniec, Luo, and Sarnak studied the one-level density distribution of the low-lying zeros of some $G L(2)$ and $G L(3)$ families of $L$-functions. Their work yields non-vanishing results conditional on the Generalized Riemann Hypothesis for these families. Therefore, let us assume the GRH throughout this section even though some other results of their paper are unconditional. For the the symmetric square family $\left\{L\left(s, \operatorname{sym}^{2} f\right) \mid f \in H_{k}\right\}$, denote a zero of $L\left(s, \operatorname{sym}^{2} f\right)$ by $\frac{1}{2}+i \gamma_{f}$, where $\gamma_{f}$ is real. Let $T$ be a slowly growing function of $k$, say $T=O(\log k)$. The number of zeros of $L\left(s, \operatorname{sym}^{2} f\right)$ counted with multiplicity with imaginary part between zero and the short height $T$ is asymptotic to $c T \log k$ for some constant $c$. Let $\tilde{\gamma}_{f}=\gamma_{f} c \log k$ so that the average spacing between these normalized zeros is 1 . Define for an even Schwartz class function $\phi$,

$$
D\left(\operatorname{sym}^{2} f, \phi\right)=\sum_{\gamma_{f}} \phi\left(\tilde{\gamma}_{f}\right) .
$$

To get an understanding of the fine behavior of the zeros near the point $s=\frac{1}{2}$, we would like to evaluate the average of $D\left(\operatorname{sym}^{2} f, \phi\right)$ over the family $H_{k}$ for $\phi$ with arbitrarily small support. Investigating this problem, Iwaniec, Luo, and Sarnak proved that for $W_{U S p}(x)=1-\frac{\sin (2 \pi x)}{2 \pi x}$, we have

$$
\frac{1}{\left|H_{k}\right|} \sum_{f \in H_{k}} D\left(\operatorname{sym}^{2} f, \phi\right) \sim \int_{\mathbb{R}} \phi(x) W_{U S p}(x) d x
$$


as $k \rightarrow \infty$ provided that $\hat{\phi}$, the Fourier transform of $\phi$, is supported on the interval $\left(-\frac{1}{2}, \frac{1}{2}\right)$. The condition on $\hat{\phi}$ is very restrictive and does not permit taking $\phi$ with finite support. The Density Conjecture claims that (1.8) holds without restriction on $\hat{\phi}$. With an extra averaging over the weight the same result is found for a larger class of test functions. Assuming the GRH for Dirichlet $L$-functions as well, the three authors proved that

$$
\frac{2}{K} \sum_{\substack{4 \leq k \leq K \\ k \equiv 0 \bmod 2}} \frac{1}{\left|H_{k}\right|} \sum_{f \in H_{k}} D\left(\operatorname{sym}^{2} f, \phi\right) \sim \int_{\mathbb{R}} \phi(x) W_{U S p}(x) d x,
$$

as $K \rightarrow \infty$ provided that $\hat{\phi}$ is supported on the interval $\left(-\frac{3}{2}, \frac{3}{2}\right)$. Notice that one expects a repulsion of zeros from the central point: when $|x| \leq \epsilon$ for a small positive constant $\epsilon$, we have $1-\frac{\sin (2 \pi x)}{2 \pi x} \ll \epsilon^{2}$ and so $\int_{-\epsilon}^{\epsilon} W_{U S p}(x) d x \ll \epsilon^{3}$.

What is amazing is that $W_{U S p}$ is the same function which occurs on the Random Matrix Theory side. A matrix $A \in U(2 N)$ has $N$ pairs of complex conjugate eigenvalues $e^{i \theta_{A}}$ on the unit circle, counted with multiplicity. Let $\tilde{\theta}_{A}=\frac{N}{\pi} \theta_{A}$ so that the average spacing between the normalized angles is 1 . Katz and Sarnak showed that for an even Schwartz class function $\phi$ we have

$$
\int_{U S p(N)} \sum_{\theta_{A}} \phi\left(\tilde{\theta}_{A}\right) d A \sim \int_{\mathbb{R}} \phi(x) W_{U S p}(x) d x,
$$

as $N \rightarrow \infty$, where $d A$ is Haar measure.

Another family that is found to be symplectic is the family of degree one quadratic Dirichlet $L$-functions $L\left(s,\left(\frac{8 d}{-}\right)\right)$ for $d$ odd and squarefree with $X \leq d \leq 2 X$. This was first shown by Özluk and Snyder [14. For another example, consider the set of degree two $L$-functions attached to 'odd' primitive cusp forms $f$ of weight 2 and prime level $q$. By odd we mean that at the central point, the functional equation of $L(s, f)$ reads $L\left(\frac{1}{2}, f\right)=-L\left(\frac{1}{2}, f\right)$. Thus in the family of such Hecke $L$-functions, it is trivial that $L\left(\frac{1}{2}, f\right)=0$. This family is found to be of type $S O(2 N+1)$, with corresponding density function

$$
W_{S O(2 N+1)}=\delta_{0}+W_{U S p(2 N)} .
$$

Above $\delta_{0}$ is the Dirac delta function, which occurs because every (unitary) matrix in $S O(2 N+1)$ has 1 as an eigenvalue. In this case it is clearly more interesting to study the non-vanishing of the derivative $L^{\prime}(s, f)$. Since $L^{\prime}(s, f)$ vanishes precisely when $L(s, f)$ has an additional zero at $s=\frac{1}{2}$, we have by the relation (1.11) that the family of derivatives is of symplectic type.

Let us now apply the density formula (1.9) to the non-vanishing of the symmetric square $L$-function at the central point. For the simple choice of $\phi(x)=\left(\frac{\sin (v \pi x)}{v \pi x}\right)^{2}$ where $v>0$, we have $\phi(0)=1, \phi(x) \geq 0$, and $\hat{\phi}$ supported on $(-v, v)$. With this test function we have from (1.9) that

$$
\frac{2}{K} \sum_{\substack{4 \leq k \leq K \\ k \equiv 0 \bmod 2}} \frac{1}{\left|H_{k}\right|} \sum_{f \in H_{k}} \operatorname{ord}_{s=\frac{1}{2}} L\left(s, \operatorname{sym}^{2} f\right) \leq \int_{\mathbb{R}} \phi(x) W_{U S p}(x) d x .
$$


Using the fact that the order of vanishing of $L\left(s, \operatorname{sym}^{2} f\right)$ at $s=\frac{1}{2}$ must be even from the sign of the functional equation, it follows that for a proportion of at least

$$
1-\frac{1}{4 v^{2}}
$$

of the family $\left\{L\left(s, \operatorname{sym}^{2} f\right) \mid f \in \bigcup_{\substack{K \leq k \leq 2 K \\ k \equiv 0}} H_{k}\right\}$ for large enough $K$ we have that $L\left(\frac{1}{2}, \operatorname{sym}^{2} f\right)$ is nonzero. Thus taking $v=\frac{3}{2}$ the proportion $\frac{8}{9}$ is gotten conditionally on GRH in [7. Actually a slightly better answer is also found by optimizing the choice of $\phi$.

Similarly for the quadratic Dirichlet $L$-function family above, by taking $v=2$ in (1.13), Özluk and Snyder conditionally showed that for large $X$ at least $\frac{15}{16}$ of the family is non-vanishing at the central point. For the third family, of odd Hecke $L$-functions, again it was conditionally obtained in [7] that when $q$ is large enough, for at least $\frac{15}{16}$ of the family we have $L^{\prime}\left(f, \frac{1}{2}\right) \neq 0$. In all three examples, the Density Conjecture implies a proportion of 1 by taking $v$ arbitrarily large.

1.3. The mollifier method. We prove Theorem 1.1 using the mollifier method, a technique which goes back to Bohr and Landau and was used by Selberg [15] to show that a positive proportion of the non-trivial zeros of the Riemann Zeta function lie on the half line. Let us begin by observing that in principle we can recover the distribution of the values $L\left(\frac{1}{2}, \operatorname{sym}^{2} f\right)$ for $f \in H_{k}$ from asymptotics for $\sum_{f \in H_{k}}^{h} L\left(\frac{1}{2}, \operatorname{sym}^{2} f\right)^{n}$ for all $n \in \mathbb{N}$. However our knowledge of these moments is very limited. For the first moment it is known (see [12]) that

$$
\sum_{f \in H_{k}}^{h} L\left(\frac{1}{2}, \operatorname{sym}^{2} f\right) \sim \log k .
$$

For the second moment we have the conjecture

Conjecture 1.4. For some constant $c$,

$$
\sum_{f \in H_{k}}^{h} L\left(\frac{1}{2}, \operatorname{sym}^{2} f\right)^{2} \sim c(\log k)^{3} .
$$

Even the upper bound $\sum_{f \in H_{k}}^{h} L\left(\frac{1}{2}, \operatorname{sym}^{2} f\right)^{2} \ll k^{\epsilon}$ is not established. The difficulty of this conjecture is what brings us to take an extra averaging over the weight. We shall show

$$
\begin{gathered}
\sum_{k \equiv 0 \bmod 2} h\left(\frac{k}{K}\right) \sum_{f \in H_{k}}^{h} L\left(\frac{1}{2}, \operatorname{sym}^{2} f\right)=K P_{h}(\log K)+O\left(K^{\epsilon}\right), \\
\sum_{k \equiv 0 \bmod 2} h\left(\frac{k}{K}\right) \sum_{f \in H_{k}}^{h} L\left(\frac{1}{2}, \operatorname{sym}^{2} f\right)^{2}=K Q_{h}(\log K)+O\left(K^{\epsilon}\right),
\end{gathered}
$$

where $P_{h}$ and $Q_{h}$ are degree one and degree three polynomials respectively which depend on $h$, and $\epsilon$ is an arbitrarily small positive constant. This shows that the average value of $L\left(\frac{1}{2}, \operatorname{sym}^{2} f\right)$ is proportional to $\log K$, and we would like to be able to conclude that a lot of values of $L\left(\frac{1}{2}, \operatorname{sym}^{2} f\right)$ are not zero. However in this way we cannot rule out the possibility of many values of $L\left(\frac{1}{2}, \operatorname{sym}^{2} f\right)$ being zero and some being very large. In fact, a comparison of the main terms of the first and second 
moments shows that there are fluctuations in the size of $L\left(\frac{1}{2}, \operatorname{sym}^{2} f\right)$. Nevertheless by the Cauchy-Schwarz inequality we get that

$$
\begin{aligned}
\sum_{k \equiv 0 \bmod 2} h\left(\frac{k}{K}\right) \sum_{\substack{f \in H_{k} \\
L\left(\frac{1}{2}, \mathrm{sym}^{2} f\right) \neq 0}}^{h} 1 & \geq \frac{\left(\sum_{k \equiv 0 \bmod 2} h\left(\frac{k}{K}\right) \sum_{f \in H_{k}}^{h} L\left(\frac{1}{2}, \mathrm{sym}^{2} f\right)\right)^{2}}{\sum_{k \equiv 0 \bmod 2} h\left(\frac{k}{K}\right) \sum_{f \in H_{k}}^{h} L\left(\frac{1}{2}, \operatorname{sym}^{2} f\right)^{2}} \\
& \gg(\log K)^{-1} K .
\end{aligned}
$$

Thus we get that for a proportion of $(\log K)^{-1}$ of Hecke cusp forms of weight less than $K$, we have $L\left(\frac{1}{2}, \operatorname{sym}^{2} f\right) \neq 0$. This however is $0 \%$ and we can improve upon it by using the mollifier method. With the power savings in the error terms of (1.16), there is room to find a little more than the second moment. Define a short Dirichlet series, called a mollifier,

$$
M(f)=\sum_{r \leq K^{a}} \frac{x_{r} a_{f}\left(r^{2}\right)}{\sqrt{r}},
$$

where $a>0$ is a constant and $x_{r}$ are coefficients to be chosen. Since the nonvanishing of $L\left(\frac{1}{2}, \operatorname{sym}^{2} f\right) M(f)$ implies the non-vanishing of $L\left(\frac{1}{2}, \operatorname{sym}^{2} f\right)$, we have as before,

$$
\sum_{k \equiv 0 \bmod 2} h\left(\frac{k}{K}\right) \sum_{\substack{f \in H_{k} \\ L\left(\frac{1}{2}, \operatorname{sym}^{2} f\right) \neq 0}}^{h} 1 \geq \frac{\left(\sum_{k \equiv 0 \bmod 2} h\left(\frac{k}{K}\right) \sum_{f \in H_{k}}^{h} L\left(\frac{1}{2}, \operatorname{sym}^{2} f\right) M(f)\right)^{2}}{\sum_{k \equiv 0 \bmod 2} h\left(\frac{k}{K}\right) \sum_{f \in H_{k}}^{h} L\left(\frac{1}{2}, \operatorname{sym}^{2} f\right)^{2} M(f)^{2}} .
$$

We shall compute the mollified moments in terms of the coefficients $x_{r}$ and then carefully choose $x_{r}$ to maximize this ratio. This choice of $M(f)$ dampens or mollifies the large values of $L\left(\frac{1}{2}, \operatorname{sym}^{2} f\right)$, so that the square of the first moment and $K$ times the second moment are comparable. Thus we will get that $L\left(\frac{1}{2}, \operatorname{sym}^{2} f\right)$ is not zero for a positive percentage of our family. In fact we will show that for an optimal mollifier of length $K^{a}$, where recall that $K^{2}$ is the size of conductor of our family, we get a proportion of $1-(1+a)^{-3}$. Our methods enable us to take $a<\frac{1}{2}$.

Soundararajan [17 proved using the mollifier method that for at least $\frac{7}{8}$ of positive odd square-free integers $X \leq d \leq 2 X$ we have $L\left(\frac{1}{2},\left(\frac{8 d}{-}\right)\right) \neq 0$ when $X$ is large enough. Taking a mollifier of length $(\sqrt{X})^{a}$, where $X$ is the size of conductor of any quadratic Dirichlet $L$-function in this family, he obtained a proportion of $1-(1+a)^{-3}$ and was able to take $a<1$ to get his result. Kowalski and Michel [11] studied the non-vanishing of $L^{\prime}\left(f, \frac{1}{2}\right)$ for odd primitive cusp forms $f$ of weight 2 and prime level $q$. Taking a mollifier of length $(\sqrt{q})^{a}$, where $q$ is the conductor of $L^{\prime}(s, f)$ and $a<\frac{1}{2}$ (which the authors remarked can be improved to $a<1$ ), they showed that for large enough $q$ we have that $L^{\prime}\left(f, \frac{1}{2}\right) \neq 0$ for a proportion of $1-(1+a)^{-3}$ of this family. Thus our result supports the belief that these three different families of $L$-functions share the same distribution of low lying zeros. In fact, from a family's symmetry type one can make predictions using the Ratios Conjecture about non-vanishing by the use of mollifiers (see 2] ).

Being able to take a mollifier $M(f)$ of length $K^{a}$ is roughly comparable to being able find the $(2+2 a)^{\text {th }}$ moment of $L\left(\frac{1}{2}, \operatorname{sym}^{2} f\right)$ on average over the weight. Taking $\hat{\phi}$ of support $(-v, v)$ in the density formula (1.9) is comparable to finding the $(2 v)^{\text {th }}$ 
moment of $L\left(\frac{1}{2}, \operatorname{sym}^{2} f\right)$ on average over the weight. Thus we can make a heuristic connection between $a$ and $v$, with $v$ corresponding to $a+1$. In this way, our proportion of non-vanishing of $\frac{19}{27}$ is the unconditional analogue of the conditional proportion $\frac{8}{9}$ of [7]. Similarly, the unconditional proportion $\frac{7}{8}$ of the other symplectic families considered above compares with the conditional proportion $\frac{15}{16}$. Notice also that the proportions $\frac{7}{8}$ and $\frac{19}{27}$ are quite good- this can be explained by the repulsion of zeros that is expected at the central point in the symplectic family. This may be compared with the orthogonal family considered in [8], where a proportion greater than $\frac{1}{2}$ is sought, but not quite achieved, to show that Landau-Siegel zeros do not exist for Dirichlet $L$-functions.

\section{THE FIRST TWISTED MOMENT}

In this section we find the first moment of $L\left(\frac{1}{2}, \operatorname{sym}^{2} f\right)$ twisted by a Fourier coeffiecient $a_{f}\left(r^{2}\right)$ on average over $k$. This will yield the first mollified moment.

Theorem 2.1. For $r \leq K^{2-\epsilon}$ we have

$$
\begin{aligned}
\sum_{k \equiv 0 \bmod 2} h\left(\frac{k-1}{K}\right) \sum_{f \in H_{k}}^{h} L\left(\frac{1}{2}, \operatorname{sym}^{2} f\right) a_{f}\left(r^{2}\right)= & \frac{K}{2 \sqrt{r}} \int_{0}^{\infty} h(u)(\log (u K / r)+C) d u \\
& +O\left(r^{\frac{1}{2}} K^{\epsilon}\right),
\end{aligned}
$$

where $C$ is an absolute constant, $\epsilon>0$ is an arbitrarily small positive constant and the error term depends on $\epsilon$ and $h$.

Let us adopt the following convention throughout this paper: $\epsilon$ will always denote an arbitrarily small positive constant, but not necessarily the same one from one occurrence to the next. Any implied constants may depend implicitly on $h$ and $\epsilon$, unless otherwise indicated. Before proving this theorem we will need some preliminary results.

2.2. Petersson trace formula. We will need the Petersson trace formula:

$$
\sum_{f \in H_{k}}^{h} a_{f}(n) a_{f}(m)=\delta_{m, n}+2 \pi i^{k} \sum_{c=1}^{\infty} \frac{S(n, m ; c)}{c} J_{k-1}\left(\frac{4 \pi \sqrt{m n}}{c}\right),
$$

where the value of $\delta_{m, n}$ is 1 if $m=n$ and 0 otherwise, $S(n, m ; c)$ is a Kloosterman sum, and $J_{k-1}$ is the $J$-Bessel function.

2.3. Approximate functional equation. While the Dirichlet series expansion of $L\left(s, \operatorname{sym}^{2} f\right)$ given in (1.2) is only valid for $\Re(s)>1$, we can use a standard tool of analytic number theory called an approximate functional equation to express $L\left(\frac{1}{2}, \operatorname{sym}^{2} f\right)$ as a weighted Dirichlet series. From property (2.4) below, the terms of this series are only significant for $n \leq k^{1+\epsilon}$, so that the length of the Dirichlet series is essentially equal to the square root of the conductor. We will use Stirling's approximation: $\log \Gamma(z)=\left(z-\frac{1}{2}\right) \log z-z+\frac{1}{2} \log \sqrt{2 \pi}+\sum_{n=1}^{m} c_{n} z^{-2 n+1}+O_{m}\left(z^{-m}\right)$ when $|\arg z|<\pi-\epsilon$ for some constants $c_{n}$.

Lemma 2.4. Approximate functional equation We have

$$
L\left(\frac{1}{2}, \operatorname{sym}^{2} f\right)=2 \sum_{n \geq 1} \frac{a_{f}\left(n^{2}\right)}{n^{\frac{1}{2}}} V_{k}(n),
$$


where for any real $\xi>0$ and $A>0$,

$$
V_{k}(\xi)=\frac{1}{2 \pi i} \int_{(A)} \frac{L_{\infty}\left(\frac{1}{2}+y\right)}{L_{\infty}\left(\frac{1}{2}\right)} \zeta(1+2 y) \xi^{-y} \frac{d y}{y}
$$

is real valued and satisfies,

$$
V_{k}(\xi) \ll_{A}\left(\frac{k}{\xi}\right)^{A}, \text { for any } A>0 .
$$

$$
V_{k}^{(B)}(\xi) \ll_{A, B} \xi^{-B}\left(\frac{k}{\xi}\right)^{A}, \text { for any } A>0 \text { and integer } B \geq 0 \text {. }
$$

$$
V_{k}(\xi)=\frac{1}{2}(\log (k / \xi)+C)+O\left(\frac{\xi}{k}\right), \text { where } C=2 \gamma-\frac{3 \log \pi}{2}-\log 2+\frac{\Gamma^{\prime}\left(\frac{3}{4}\right)}{2 \Gamma\left(\frac{3}{4}\right)} .
$$

We also have that

$$
V_{k}(\xi)=\frac{1}{2 \pi i} \int_{(A)} G(y) \frac{\zeta(1+2 y)}{y}\left(\frac{k}{\xi}\right)^{y} d y+O\left(\xi^{-\epsilon} k^{-1+\epsilon}\right),
$$

for any $A>0$, where $G(y)=\pi^{-\frac{3}{2} y} 2^{-y} \frac{\Gamma\left(\frac{y}{2}+\frac{3}{4}\right)}{\Gamma\left(\frac{3}{4}\right)}$ exponentially decays on vertical lines.

Proof. Define for $-\frac{1}{2} \leq c \leq \frac{3}{2}$,

$$
I(c)=\frac{1}{2 \pi i} \int_{(c)} L\left(\frac{1}{2}+y, \operatorname{sym}^{2} f\right) \frac{L_{\infty}\left(\frac{1}{2}+y\right)}{L_{\infty}\left(\frac{1}{2}\right)} \frac{d y}{y},
$$

where the integral is taken from $c-i \infty$ to $c+i \infty$ and converges absolutely because the integrand decays exponentially as $|\Im(y)| \rightarrow \infty$. In the range $\Re(y) \geq-\frac{1}{2}$ the integrand has a simple pole at $y=0$ with residue $L\left(\frac{1}{2}, \operatorname{sym}^{2} f\right)$. Thus by Cauchy's theorem we have $I\left(\frac{3}{2}\right)-I\left(-\frac{1}{2}\right)=L\left(\frac{1}{2}, \operatorname{sym}^{2} f\right)$. Using the functional equation (1.4) and a change of variables we have $I\left(-\frac{1}{2}\right)=-I\left(\frac{1}{2}\right)$ and so we get that $L\left(\frac{1}{2}, \operatorname{sym}^{2} f\right)=I\left(\frac{3}{2}\right)+I\left(\frac{1}{2}\right)=2 I\left(\frac{3}{2}\right)$. At $\Re(y)=\frac{3}{2}$ we can use the Dirichlet series expansion (1.2) to get

$$
I\left(\frac{3}{2}\right)=\sum_{n \geq 1} \frac{a\left(n^{2}\right)}{n^{\frac{1}{2}}} \int_{(3 / 2)} \frac{\zeta\left(2\left(\frac{1}{2}+y\right)\right) L_{\infty}\left(\frac{1}{2}+y\right)}{n^{y} L_{\infty}\left(\frac{1}{2}\right)} \frac{d y}{y}=\sum_{n \geq 1} \frac{a\left(n^{2}\right)}{n^{\frac{1}{2}}} V_{k}(n),
$$

where we implicity exchanged summation and integration by absolute convergence. This establishes (2.2).

For $\Re(y)=A$ we have by Stirling's estimates that

$$
\left|\frac{L_{\infty}\left(\frac{1}{2}+y\right)}{L_{\infty}\left(\frac{1}{2}\right)}\right| \ll\left|\Gamma\left(\frac{3+2 y}{4}\right)\right| \frac{\Gamma\left(k+A-\frac{1}{2}\right)}{\Gamma\left(k-\frac{1}{2}\right)} \ll_{A}\left|\Gamma\left(\frac{3+2 y}{4}\right)\right| k^{A} .
$$

This implies (2.4). We obtain (2.5) by first differentiating $V_{k}(\xi)$ and then using Stirling's estimates. To get (2.6) we move the line of integration of $V_{k}(\xi)$ to $\Re(y)=$ -1 , crossing a double pole at $y=0$. The main term (2.6) is the residue from this pole and the error is given by Stirling's estimates. 
Let us turn to (2.7). We have $\frac{L_{\infty}\left(\frac{1}{2}+y\right)}{L_{\infty}\left(\frac{1}{2}\right)}=G(y) \frac{\Gamma\left(y+k-\frac{1}{2}\right)}{\Gamma\left(k-\frac{1}{2}\right)}$. For $\Im(y) \leq k^{\epsilon}$ it follows from Stirling's estimates that $\frac{\Gamma\left(y+k-\frac{1}{2}\right)}{\Gamma\left(k-\frac{1}{2}\right)}=k^{y}\left(1+O_{A}\left(k^{-1+\epsilon}\right)\right)=k^{y}+O_{A}\left(k^{A-1+\epsilon}\right)$. Since $\left|\frac{\Gamma\left(y+k-\frac{1}{2}\right)}{\Gamma\left(k-\frac{1}{2}\right)}\right| \leq \frac{\Gamma\left(A+k-\frac{1}{2}\right)}{\Gamma\left(k-\frac{1}{2}\right)} \ll_{A} k^{A}$ and $G(y)$ is exponentially decreasing on vertical strips, we can restrict the integral in $V_{k}(\xi)$ to $\Im(y) \leq k^{\epsilon}$ with an error of $O_{A}\left((k / \xi)^{A} e^{-k^{\epsilon}}\right)$. Taking $A=\epsilon$ we get $V_{k}(\xi)=\frac{1}{2 \pi i} \int_{(\epsilon)} G(y) \frac{\zeta(1+2 y)}{y}\left(\frac{k}{\xi}\right)^{y} d y+$ $O\left(\xi^{-\epsilon} k^{-1+\epsilon}\right)$. The line of integration can be moved any $A>0$ to get (2.7).

2.5. An average of the $J$-Bessel function. We have the following standard estimates: $\left|J_{k-1}(t)\right| \leq 1$ for all $t \geq 0, J_{k-1}(t) \sim \frac{1}{\Gamma(k)}(t / 2)^{k-1}$ for $t \leq k^{1 / 2-\epsilon}$ and $J_{k-1}(t) \sim\left(\frac{\pi}{2} t\right)^{-1 / 2} \cos \left(t-\frac{\pi}{2} k+\frac{\pi}{4}\right)$ for $t \geq k^{2+\epsilon}$. The long term behavior of the $J$-Bessel function manifests itself when we average over $k$ :

Lemma 2.6. We have for $t>0$,

$$
\begin{aligned}
\sum_{k \equiv 0 \bmod 2} 2 i^{k} h\left(\frac{k-1}{K}\right) & J_{k-1}(t) \\
& =-\frac{K}{\sqrt{t}} \Im\left(e^{-2 \pi i / 8} e^{i t} \hbar\left(\frac{K^{2}}{2 t}\right)\right)+O\left(\frac{t}{K^{4}} \int_{-\infty}^{\infty} v^{4}|\hat{h}(v)| d v\right),
\end{aligned}
$$

where $\hbar(v)=\int_{0}^{\infty} \frac{h(\sqrt{u})}{\sqrt{2 \pi u}} e^{i u v} d u$ and $\hat{h}$ denotes the Fourier transform of $h$. The implied constant is absolute.

Proof. We refer to [6, Lemma 5.8] for the full proof. It is shown there that the sum we want equals

$$
\begin{aligned}
& 2 \sum_{k \equiv 0 \bmod 4} h\left(\frac{k-1}{K}\right) J_{k-1}(t)-2 \sum_{k \equiv 2 \bmod 4} h\left(\frac{k-1}{K}\right) J_{k-1}(t) \\
& =-2 \int_{-\infty}^{\infty} K \hat{h}(K v) \sin (t \cos 2 \pi v) d v \\
& =-2 \int_{-\infty}^{\infty} K \hat{h}(K v) \sin \left(t-2 \pi^{2} t v^{2}\right) d v+O\left(\int_{-\infty}^{\infty} t v^{4} K|\hat{h}(K v)| d v\right),
\end{aligned}
$$

where the last line follows by writing $\cos 2 \pi v=1-2 \pi^{2} v^{2}+O\left(v^{4}\right)$. We have written the main term as it appears in [7, Corollary 8.2].

We have that $\hbar(v) \ll 1$ and by integrating by parts several times we get that $\hbar(v) \ll_{B} v^{-B}$ for any $B \geq 0$. Thus the main term of (2.11) is not dominant if $t \leq K^{2-\epsilon}$.

Define

$$
W_{K}(n, m, v)=\int_{0}^{\infty} \frac{V_{\sqrt{u} K+1}(n) V_{\sqrt{u} K+1}(m) h(\sqrt{u})}{\sqrt{2 \pi u}} e^{i u v} d u .
$$


By lemma 2.6 we have

$$
\begin{aligned}
\sum_{k \equiv 0 \bmod 2} 2 i^{k} h\left(\frac{k-1}{K}\right) & V_{k}(n) V_{k}(m) J_{k-1}(t)=-\frac{K}{\sqrt{t}} \Im\left(e^{-2 \pi i / 8} e^{i t} W_{K}\left(n, m, \frac{K^{2}}{2 t}\right)\right) \\
+ & O\left(\frac{t}{K^{4}} \int_{-\infty}^{\infty} v^{4}\left|\int_{0}^{\infty} V_{u K+1}(n) V_{u K+1}(m) h(u) e^{i u v} d u\right| d v\right) .
\end{aligned}
$$

Using the integral definition of $V_{k}(n)$ we have

$$
\begin{aligned}
W_{K}(n, m, v)= & \frac{1}{(2 \pi i)^{2}} \int_{\left(A_{1}\right)} \int_{\left(A_{2}\right)} G(y) G(x) \frac{\zeta(1+2 y)}{y} \frac{\zeta(1+2 x)}{x} \frac{1}{m^{x} n^{y}} \\
& \int_{0}^{\infty} \frac{\Gamma\left(\sqrt{u} K+y+\frac{1}{2}\right)}{\Gamma\left(\sqrt{u} K+\frac{1}{2}\right)} \frac{\Gamma\left(\sqrt{u} K+x+\frac{1}{2}\right)}{\Gamma\left(\sqrt{u} K+\frac{1}{2}\right)} \frac{h(\sqrt{u})}{\sqrt{2 \pi u}} e^{i u v} d u d y d x
\end{aligned}
$$

for any $A_{1}, A_{2}>0$. By integrating by parts several times and Stirling's formula we have for $\Re(y)=A_{2},|y| \leq K^{\epsilon}, \Re(x)=A_{1}$ and $|x| \leq K^{\epsilon}$ that

$$
\begin{aligned}
\int_{0}^{\infty} \frac{\Gamma\left(\sqrt{u} K+y+\frac{1}{2}\right)}{\Gamma\left(\sqrt{u} K+\frac{1}{2}\right)} \frac{\Gamma\left(\sqrt{u} K+x+\frac{1}{2}\right)}{\Gamma\left(\sqrt{u} K+\frac{1}{2}\right)} & \frac{h(\sqrt{u})}{\sqrt{2 \pi u}} e^{i u v} d u \\
& \ll_{B, A_{1}, A_{2}} \frac{(1+|x|+|y|)^{B} K^{A_{1}+A_{2}}}{v^{B}},
\end{aligned}
$$

for any $B \geq 0$. This together with the fact that $G(y)$ decreases exponentially as $\Im(y) \rightarrow \infty$ implies

$$
W_{K}(n, m, v) \ll_{B, A_{1}, A_{2}}\left(\frac{K}{n}\right)^{A_{1}}\left(\frac{K}{m}\right)^{A_{2}} v^{-B} .
$$

Thus $W$ is essentially supported on $n \leq K^{1+\epsilon}, m \leq K^{1+\epsilon}$, and $v \leq K^{\epsilon}$. Similarly by integrating by parts, the error in (2.14) is

$$
O_{A_{1}, A_{2}}\left(\frac{t}{K^{4}}\left(\frac{K}{n}\right)^{A_{1}}\left(\frac{K}{m}\right)^{A_{2}}\right)
$$

for any $A_{1}, A_{2}>0$. In applications $t$ will be bounded by a power of $K$ and so this error term essentially only appears when $n \leq K^{1+\epsilon}$ and $m \leq K^{1+\epsilon}$.

In combination with (2.7) we have

$$
W_{K}(n, m, v)=W\left(\frac{n}{K}, \frac{m}{K}, v\right)+O\left(K^{-1+\epsilon}\right),
$$

where we define for real $\xi_{1}>0$ and $\xi_{2}>0$,

$W\left(\xi_{1}, \xi_{2}, v\right)=\frac{1}{(2 \pi i)^{2}} \int_{\left(A_{1}\right)} \int_{\left(A_{2}\right)} G(y) G(x) \frac{\zeta(1+2 y)}{y} \frac{\zeta(1+2 x)}{x} \xi_{1}^{-y} \xi_{2}^{-x} \hbar_{x+y}(v) d y d x$

and

$$
\hbar_{z}(v)=\int_{0}^{\infty} \frac{h(\sqrt{u})}{\sqrt{2 \pi u}} u^{z / 2} e^{i u v} d u,
$$

for a complex number $z$. By integration by parts we have

$$
\hbar_{z}(v) \ll_{\Re(z), B}(1+|z|)^{B} v^{-B}
$$

and

$$
W\left(\xi_{1}, \xi_{2}, v\right) \ll_{B, A_{1}, A_{2}} \xi_{1}^{-A_{1}} \xi_{2}^{-A_{2}} v^{-B},
$$


for $A_{1}, A_{2}>0$ and $B \geq 0$.

Similarly we have

$$
\begin{aligned}
\sum_{k \equiv 0 \bmod 2} 2 i^{k} h\left(\frac{k-1}{K}\right) V_{k}(n) J_{k-1}(t)=- & \frac{K}{\sqrt{t}} \Im\left(e^{-2 \pi i / 8} e^{i t} W_{K}\left(n, \frac{K^{2}}{2 t}\right)\right) \\
& +O_{A}\left(\frac{t}{K^{4}}\left(\frac{K}{n}\right)^{A}\right),
\end{aligned}
$$

where $W_{K}(n, v)=\int_{0}^{\infty} \frac{V_{\sqrt{u} K+1}(n) h(\sqrt{u})}{\sqrt{2 \pi u}} e^{i u v} d u$ and $A>0$. We have

$$
W_{K}(n, v) \ll_{A, B}\left(\frac{K}{n}\right)^{A} v^{-B},
$$

for any $A>0$ and $B \geq 0$.

2.7. Kloosterman sums. We will use Weil's bound for the Kloosterman sum,

$$
|S(n, m, c)| \leq d(c) c^{\frac{1}{2}}(n, m, c)^{\frac{1}{2}},
$$

and the average bound which follows from this,

$$
\sum_{n \leq x}\left|S\left(n^{2}, m^{2}, c\right)\right| \ll c^{\frac{1}{2}+\epsilon} \sum_{n \leq x}\left(n^{2}, c\right)^{\frac{1}{2}} \ll c^{\frac{1}{2}+\epsilon} \sum_{\alpha \mid c} \sum_{\substack{n \leq x \\(n, c)=\alpha}} \alpha \ll x c^{\frac{1}{2}+\epsilon} .
$$

2.8. Proof of Theorem 2.1. Using the approximate functional equation and the Petersson trace formula we get that the first twisted moment is

$$
\begin{aligned}
& \sum_{k \equiv 0 \bmod 2} h\left(\frac{k-1}{K}\right) \sum_{f \in H_{k}}^{h} L\left(\frac{1}{2}, \operatorname{sym}^{2} f\right) a_{f}\left(r^{2}\right) \\
& =2 \sum_{k \equiv 0 \bmod 2} h\left(\frac{k-1}{K}\right)\left(\frac{V_{k}(r)}{\sqrt{r}}+\sum_{n, c \geq 1} 2 \pi i^{k} \frac{S\left(n^{2}, r^{2}, c\right)}{c} J_{k-1}\left(\frac{4 \pi n r}{c}\right) \frac{V_{k}(n)}{\sqrt{n}}\right) .
\end{aligned}
$$

Estimating $V_{k}(r)$ using (2.6) and using (2.24) we get that (2.28) equals,

$$
\begin{aligned}
\sum_{k \equiv 0 \bmod 2} h\left(\frac{k-1}{K}\right) \frac{\log (k / r)+C+O(r / K)}{\sqrt{r}} & \\
-2 \pi \sum_{n \geq 1} \frac{1}{\sqrt{n}} K \sum_{c \geq 1} \Im(e(-1 / 8) & \left.\frac{1}{\sqrt{4 \pi n r / c}} e(2 n r / c) \frac{S\left(n^{2}, r^{2}, c\right)}{c} W_{K}\left(n, \frac{K^{2} c}{8 \pi n}\right)\right) \\
& +O\left(K^{-4+\epsilon} \sum_{n, c \geq 1} r \sqrt{n} \frac{\left|S\left(n^{2}, r^{2}, c\right)\right|}{c^{2}}\left(\frac{K}{n}\right)^{A}\right),
\end{aligned}
$$

for any $A>0$. In the third line of (2.29), the sum can be restricted to $n \leq K^{1+\epsilon}$ with an error of $O\left(K^{-10}\right)$. By the bound (2.25) on $W_{K}$, the sums in the second line can be restricted to $n \leq K^{1+\epsilon}$ and $c \leq n r / K^{2-\epsilon}$, with an error of $O\left(K^{-10}\right)$. Then using Weil's bound for the Kloosterman sum, we find that the last two lines of (2.29) are bounded by $O\left(r^{\frac{1}{2}} K^{\epsilon}\right)$. The error in the first line of (2.29) is also $O\left(r^{\frac{1}{2}} K^{\epsilon}\right)$. Finally for the main term, note that by Poisson summation we have

$$
\sum_{k \equiv 0 \bmod 2} h\left(\frac{k-1}{K}\right)=\frac{K}{2} \hat{h}(0)+O_{B}\left(K^{-B}\right),
$$


for any $B>0$, where $\hat{h}$ denotes the Fourier transform of $h$. Writing $\log k=$ $\log \left(\frac{k-1}{K} K\right)+O\left(k^{-1}\right)$ and using (2.30) the proof is complete.

\section{The SECOND TWISTED MOMENT}

In this section we find the second moment of $L\left(\frac{1}{2}, \operatorname{sym}^{2} f\right)$ twisted by a Fourier coeffiecient $a_{f}\left(r^{2}\right)$ on average over $k$. This will yield the second mollified moment. Interesting features make this computation natural and simple.

Theorem 3.1. For any positive integer $d$, write $d=d_{1} d_{2}^{2}$ with $d_{1}$ square-free. For $r \leq K^{1-\epsilon}$ we have

$$
\begin{aligned}
& \sum_{k \equiv 0 \bmod 2} h\left(\frac{k-1}{K}\right) \sum_{f \in H_{k}}^{h} L\left(\frac{1}{2}, \operatorname{sym}^{2} f\right)^{2} a_{f}\left(r^{2}\right)= \\
& \begin{aligned}
\frac{K}{\sqrt{r}} \sum_{d \mid r^{2}} \frac{1}{\sqrt{d_{1}}} \int_{0}^{\infty} h(u)\left(\frac{1}{4} \log ^{2} \frac{u K}{d_{1} d_{2}} \log \frac{u K d_{2}}{r}-\frac{1}{12} \log ^{3} \frac{u K}{d_{1} d_{2}}+P_{2}\left(\log \frac{u K}{d_{1} d_{2}}\right)\right. \\
\left.+\log \frac{u K d_{2}}{r} P_{1}\left(\log \frac{u K}{d_{1} d_{2}}\right)\right) d u+O\left(r^{\frac{1}{2}} K^{\epsilon}\right) .
\end{aligned}
\end{aligned}
$$

where $P_{i}$ is a polynomial of degree $i$ and we write $\log ^{i} x=(\log x)^{i}$.

We will need the following results.

3.2. Sum of Kloosterman sums. We will need the following sum of twisted Kloosterman sums.

Lemma 3.3. Suppose $n, b, d$ and $r$ are positive integers with $r \leq K^{1-\epsilon}$. We have

$$
\begin{aligned}
\sum_{n \geq 1} S\left(n^{2}, b^{2}, c\right) e(2 n b / c) n^{-1} W\left(\frac{n}{K}, \frac{b d}{r K}, \frac{K^{2} c}{8 \pi n b}\right) \\
\quad= \begin{cases}\phi(c) c^{-\frac{1}{2}} \sum_{n \geq 1} n^{-1} W\left(\frac{n}{K}, \frac{b d}{r K}, \frac{K^{2} c}{8 \pi n b}\right)+O\left(K^{-100}\right) & \text { if } c \text { is a square, } \\
O\left(K^{-100}\right) & \text { otherwise. }\end{cases}
\end{aligned}
$$

Proof. We have

$$
\sum_{n \geq 1} S\left(n^{2}, b^{2}, c\right) e(2 n b / c) n^{-1} W\left(\frac{n}{K}, \frac{b d}{r K}, \frac{K^{2} c}{8 \pi n b}\right)
$$

$$
=\sum_{a \bmod c} S\left(a^{2}, b^{2}, c\right) e(2 a b / c) \sum_{\substack{n \geq 1 \\ n \equiv a \bmod c}} n^{-1} W\left(\frac{n}{K}, \frac{b d}{r K}, \frac{K^{2} c}{8 \pi n b}\right) .
$$

We first deal with the inner sum above. We assume throughout this proof that $b \leq r K^{1+\epsilon / 4} \leq K^{2-\epsilon / 2}$ and $c \leq n b K^{-2+\epsilon / 4} \leq K^{1-\epsilon / 2}$, since otherwise both sides of (3.1) would be of size $O\left(K^{-100}\right)$. This follows by the bound (2.23). Using definition (2.20) and integration by parts we have have the following bound on the derivatives of $W$ for $\xi>0$ :

$$
\frac{\partial^{j}}{\partial \xi^{j}} W\left(\xi, \frac{b d}{r K}, \frac{K c}{8 \pi \xi b}\right) \ll_{j, A, B} \xi^{-j-A}\left(\frac{\xi b}{K c}\right)^{B} K^{\epsilon},
$$

for any $A>0, B \geq 0$ and integer $j \geq 0$. We extend the definition of $W\left(\xi, \frac{b d}{r K}, \frac{K c}{8 \pi \xi b}\right)$ to all real numbers $\xi$ by setting $W\left(\xi, \frac{b d}{r K}, \frac{K c}{8 \pi \xi b}\right)=0$ for $\xi \leq 0$; by the bound just 
shown this is still a smooth function of $\xi$. Now by Poisson summation we have that the inner sum of (3.2) equals

$$
\begin{aligned}
& \sum_{n \equiv a \bmod c} n^{-1} W\left(\frac{n}{K}, \frac{b d}{r K}, \frac{K^{2} c}{8 \pi n b}\right) \\
& =\frac{1}{c} \sum_{n} \int_{-\infty}^{\infty} \xi^{-1} W\left(\xi, \frac{b d}{r K}, \frac{K c}{8 \pi \xi b}\right) e^{\frac{2 \pi i K}{c} \xi n} e^{\frac{-2 \pi i a}{c} n} d \xi .
\end{aligned}
$$

By integrating by parts $j$ times and using (3.3) we find that for $|n| \neq 0$ we have

$$
\int_{-\infty}^{\infty} \xi^{-1} W\left(\xi, \frac{b d}{r K}, \frac{K c}{8 \pi \xi b}\right) e^{\frac{2 \pi i K}{c} \xi n} e^{\frac{-2 \pi i a}{c} n} d \xi \ll_{j}\left(\frac{c}{K|n|}\right)^{j}\left(\frac{b}{K c}\right)^{j} K^{10} .
$$

Since $b \leq K^{2-\epsilon / 2}$, the above is of size $O\left(|n|^{-2} K^{-100}\right)$ by taking $j$ large enough. Thus only the $n=0$ term is significant and (3.4) equals

$$
\frac{1}{c} \int_{-\infty}^{\infty} \xi^{-1} W\left(\xi, \frac{b d}{r K}, \frac{K c}{8 \pi \xi b}\right) d \xi+O\left(K^{-100}\right)=\frac{1}{c} \sum_{n \geq 1} n^{-1} W\left(\frac{n}{K}, \frac{b d}{r K}, \frac{K^{2} c}{8 \pi n b}\right)+\left(K^{-100}\right),
$$

by Poisson summation again.

As for the outer sum of (3.2), opening the Kloosterman sum we have

$$
\begin{aligned}
& \sum_{a \bmod c} S\left(a^{2}, b^{2}, c\right) e(2 a b / c)= \sum_{\substack{a, \gamma \bmod c \\
(\gamma, c)=1}} e\left(\frac{a^{2} \bar{\gamma}+b^{2} \gamma+2 a b}{c}\right) \\
&=\sum_{\substack{a, \gamma \bmod c \\
(\gamma, c)=1}} e\left(\frac{\gamma(a+b)^{2}}{c}\right)=\sum_{\substack{a, \gamma \bmod c \\
(\gamma, c)=1}} e\left(\frac{\gamma a^{2}}{c}\right)=\sum_{a \bmod c} r_{c}\left(a^{2}\right),
\end{aligned}
$$

where $r_{c}\left(a^{2}\right)$ is a Ramanujan sum and $\bar{\gamma} \gamma \equiv 1 \bmod c$. We get the last line above by replacing $a$ by $a \gamma$, then $a$ by $a-b$. Since $r_{c}(n)=\mu\left(\frac{c}{(n, c)}\right) \phi(c) / \phi\left(\frac{c}{(n, c)}\right)$, we have that the sum of Ramanujan sums in (3.7) is

$$
\phi(c) \sum_{a \bmod c} \mu\left(\frac{c}{\left(a^{2}, c\right)}\right) / \phi\left(\frac{c}{\left(a^{2}, c\right)}\right) .
$$

This is multiplicative in $c$, and so we can assume $c$ to be a prime power $p^{k}$. Let $\operatorname{ord}_{p}(a)$ denote the highest power of $p$ dividing $a$. If $k$ is even, $\mu\left(\frac{c}{\left(a^{2}, c\right)}\right)=0$ if $\operatorname{ord}_{p}(a)<k / 2$, while otherwise $\mu\left(\frac{c}{\left(a^{2}, c\right)}\right) / \phi\left(\frac{c}{\left(a^{2}, c\right)}\right)=1$. So if $k$ is even, (3.8) equals $\phi(c) p^{k} / p^{k / 2}=\phi(c) \sqrt{c}$. For $k$ odd, if $\operatorname{ord}_{p}(a)<(k-1) / 2$ then $\mu\left(\frac{c}{\left(a^{2}, c\right)}\right)=0$, if $\operatorname{ord}_{p}(a)=(k-1) / 2$ then $\mu\left(\frac{c}{\left(a^{2}, c\right)}\right) / \phi\left(\frac{c}{\left(a^{2}, c\right)}\right)=-\frac{1}{p-1}$, and if $\operatorname{ord}_{p}(a)>(k-$ 1) $/ 2$ then $\mu\left(\frac{c}{\left(a^{2}, c\right)}\right) / \phi\left(\frac{c}{\left(a^{2}, c\right)}\right)=1$. So if $k$ is odd, (3.8) equals $\phi(c) p^{k} / p^{(k+1) / 2}-$ $\phi(c) \frac{1}{p-1}\left(p^{k} / p^{(k-1) / 2}-p^{k} / p^{(k+1) / 2}\right)=0$.

3.4. A Mellin transform. Denote by $\tilde{f}$ the Mellin transform of a function $f$.

Lemma 3.5. For $0<\Re(s)<1$, we have

$$
\widetilde{\hbar}_{z}(s)=\int_{0}^{\infty} \frac{h(\sqrt{u})}{\sqrt{2 \pi u}} u^{z / 2-s} \Gamma(s)(\cos (s \pi / 2)+i \sin (s \pi / 2)) d u
$$


and the bound $\widetilde{\hbar}_{z}(s) \ll_{\Re(z)}(1+|z|)^{3}|s|^{-2}$. For $0<c<1$ we have

$$
\hbar_{z}(v)=\frac{1}{2 \pi i} \int_{(c)} v^{-s} \widetilde{\hbar}_{z}(s) d s .
$$

Proof. We have the Mellin transform $\int_{0}^{\infty} v^{s-1} e^{i v} d v=\Gamma(s) e^{i \pi s / 2}$ for $0<\Re(s)<1$. Thus since $h$ is compactly supported on $(0, \infty)$ we have

$$
\int_{0}^{T} v^{s-1} \int_{0}^{\infty} \frac{h(\sqrt{u})}{\sqrt{2 \pi u}} u^{z / 2} e^{i u v} d u d v=\int_{0}^{\infty} \frac{h(\sqrt{u})}{\sqrt{2 \pi u}} u^{z / 2}\left(u^{-s} \Gamma(s) e^{i \pi s / 2}+o_{s}(1)\right) d u
$$

as $T \rightarrow \infty$. This gives (3.9). By Stirling's approximation we have $\Gamma(s) e^{i \pi s / 2} \ll|s|$ for $0<\Re(s)<1$. So on integrating by parts the expression (3.9) we get

$$
\widetilde{\hbar}_{z}(s) \ll_{\Re(z)}(1+|z|)^{3}|s|^{-2} .
$$

By the bound (2.22) we have that $\int_{0}^{\infty} v^{s-1} \hbar_{z}(v) d v$ converges absolutely, so that (3.10) follows by Mellin inversion.

3.6. Proof of Theorem 3.1; the diagonal. Recall the Hecke multiplicative property

$$
a_{f}\left(m^{2}\right) a_{f}\left(r^{2}\right)=\sum_{d \mid\left(m^{2}, r^{2}\right)} a_{f}\left(m^{2} r^{2} / d^{2}\right) .
$$

Using the approximate functional equation and the Petersson trace formula, we get that the twisted second moment is

$$
\sum_{k \equiv 0 \bmod 2} h\left(\frac{k-1}{K}\right) \sum_{f \in H_{k}}^{h} L\left(\frac{1}{2}, \operatorname{sym}^{2} f\right)^{2} a_{f}\left(r^{2}\right)
$$

$$
\begin{gathered}
=4 \sum_{k \equiv 0 \bmod 2} h\left(\frac{k-1}{K}\right) \sum_{m \geq 1} \sum_{d \mid\left(r^{2}, m^{2}\right)} \sum_{\substack{n \geq 1 \\
n=m r / d}} \frac{1}{\sqrt{n m}} V_{k}(n) V_{k}(m) \\
+4 \sum_{k \equiv 0 \bmod 2} h\left(\frac{k-1}{K}\right) \sum_{n, m \geq 1} \sum_{d \mid\left(r^{2}, m^{2}\right)} 2 \pi i^{k} \frac{S\left(n^{2}, m^{2} r^{2} / d^{2}, c\right)}{c} J_{k-1}\left(\frac{4 \pi n m r / d}{c}\right) \\
\times \frac{V_{k}(n) V_{k}(m)}{\sqrt{n m}} .
\end{gathered}
$$

Write $d=d_{1} d_{2}^{2}$, with $d_{1}$ square-free, and note that $d \mid m^{2}$ if and only if $d_{1} d_{2} \mid m$. Thus we may replace $m$ above by $m d_{1} d_{2}$ and get that (3.14) equals

$$
\begin{gathered}
=4 \sum_{k \equiv 0 \bmod 2} h\left(\frac{k-1}{K}\right) \sum_{d \mid r^{2}} \sum_{m \geq 1} \frac{1}{m \sqrt{r d_{1}}} V_{k}\left(m r / d_{2}\right) V_{k}\left(m d_{1} d_{2}\right) \\
+4 \sum_{k \equiv 0 \bmod 2} h\left(\frac{k-1}{K}\right) \sum_{d \mid r^{2}} \sum_{c, n, m \geq 1} 2 \pi i^{k} \frac{S\left(n^{2}, m^{2} r^{2} / d_{2}^{2}, c\right)}{c} J_{k-1}\left(\frac{4 \pi n m r / d_{2}}{c}\right) \\
\times \frac{V_{k}(n) V_{k}\left(m d_{1} d_{2}\right)}{\sqrt{n m d_{1} d_{2}}} .
\end{gathered}
$$


We first evaluate line (3.15), the diagonal contribution to the second moment, and turn to the off-diagonal part (3.16) in the next section. We have using (2.7),

$$
\sum_{m \geq 1} \frac{V_{k}\left(m r / d_{2}\right) V_{k}\left(m d_{1} d_{2}\right)}{m}
$$

$$
\begin{array}{r}
=\frac{1}{(2 \pi i)^{2}} \int_{(\epsilon)} \int_{(\epsilon)} \frac{G(x) G(y) \zeta(1+2 x) \zeta(1+2 y)}{x y} \frac{k^{x+y}}{\left(r / d_{2}\right)^{x}\left(d_{1} d_{2}\right)^{y}} \zeta(1+x+y) d x d y \\
+O\left(K^{-1+\epsilon}\right) .
\end{array}
$$

We move the line of integration $\Re(x)=\epsilon$ to $\Re(x)=-1+\epsilon$, crossing a double pole at $x=0$ and a simple pole at $x=-y$. The integral on the new line is $\ll r K^{-1+\epsilon}$. Thus since $\zeta(1+2 x) / x=1 /\left(2 x^{2}\right)+\gamma / x+\ldots$ we get that (3.17) is

$$
\frac{1}{2 \pi i} \int_{(\epsilon)} \frac{G(y) \zeta(1+2 y)}{y} \frac{k^{y}}{\left(d_{1} d_{2}\right)^{y}}\left(\left(\gamma+\frac{G^{\prime}(0)}{2}+\frac{\log \left(k d_{2} / r\right)}{2}\right) \zeta(1+y)+\frac{\zeta^{\prime}(1+y)}{2}\right) d y
$$

$$
+\frac{1}{2 \pi i} \int_{(\epsilon)} \frac{G(-y) G(y) \zeta(1-2 y) \zeta(1+2 y)}{-y^{2}} \frac{r^{y}}{d^{y}} d y+O\left(r K^{-1+\epsilon}\right) .
$$

Now we move the line of integration in (3.18) to $\Re(y)=-1+\epsilon$, crossing a pole of order 4 at $y=0$. The integral on the new line is $\ll\left(K / d_{1} d_{2}\right)^{-1+\epsilon}$. From the residue at the pole we find that (3.18) equals

$$
\begin{array}{r}
\frac{1}{8}\left(\log \frac{k}{d_{1} d_{2}}\right)^{2} \log \frac{k d_{2}}{r}-\frac{1}{24}\left(\log \frac{k}{d_{1} d_{2}}\right)^{3}+2 P_{2}\left(\log \frac{k}{d_{1} d_{2}}\right)+2 \log \frac{k d_{2}}{r} P_{1}\left(\log \frac{k}{d_{1} d_{2}}\right) \\
+O\left(r K^{-1+\epsilon}\right),
\end{array}
$$

where $P_{i}$ is a polynomial of degree $i$. Inserting (3.20) into (3.15) and averaging over $k$ using (2.30) gives the main term of Theorem 3.1.

Inserting (3.19) into (3.15) gives that the contribution of this term to the diagonal is

$$
\frac{-2 K}{\sqrt{r}} \hat{h}(0) \frac{1}{2 \pi i} \int_{(\epsilon)} \frac{G(-y) G(y) \zeta(1-2 y) \zeta(1+2 y)}{y^{2}} \sum_{d \mid r^{2}} \frac{1}{\sqrt{d_{1}}} \frac{r^{y}}{d^{y}} d y+O\left(r K^{\epsilon}\right)
$$

We will see that this cancels out with the off-diagonal! Note that the integrand is an even function of $y$. One can check that $\sum_{d \mid r^{2}} \frac{1}{\sqrt{d_{1}}} \frac{r^{y}}{d y}$ is even by observing that it is multiplicative in $r$ and then checking that it is even for $r$ equal to a prime power.

3.7. Proof of Theorem 3.1; the off-diagonal. In this section we evaluate line (3.16). By (2.14) we have that (3.16) equals

$$
\begin{gathered}
-4 \pi K \sum_{d \mid r^{2}} \sum_{n, m, c \geq 1} \frac{S\left(n^{2}, m^{2} r^{2} / d_{2}^{2}, c\right)}{c \sqrt{n m d_{1} d_{2}}} \Im\left(\frac{e\left(2 n m r / c d_{2}-1 / 8\right)}{\sqrt{4 \pi n m r / c d_{2}}} W_{K}\left(n, m d_{1} d_{2}, \frac{K^{2} c d_{2}}{8 \pi n m r}\right)\right) \\
+O\left(K^{-4} \sum_{d \mid r^{2}} \sum_{n, m, c \geq 1} r \sqrt{n m} \frac{\left|S\left(n^{2}, m^{2} r^{2} / d_{2}^{2}, c\right)\right|}{c^{2}}\left(\frac{K}{n}\right)^{A_{1}}\left(\frac{K}{m}\right)^{A_{2}}\right) .
\end{gathered}
$$


RIZWANUR KHAN

The error term above is $O\left(r K^{-1+\epsilon}\right)$ by restricting the summation to $n, m \leq K^{1+\epsilon}$ and using Weil's bound for the Kloosterman sum. The main term equals by (2.17), (2.19) and (2.23),

$$
\begin{aligned}
-K \frac{2 \sqrt{\pi}}{\sqrt{r}} \Im\left(\sum_{d \mid r^{2}} \sum_{n, m, c \geq 1}\right. & \left.\frac{S\left(n^{2}, m^{2} r^{2} / d_{2}^{2}, c\right)}{n m \sqrt{c d_{1}}} e\left(2 n m r / c d_{2}-1 / 8\right) W\left(\frac{n}{K}, \frac{m d_{1} d_{2}}{K}, \frac{K^{2} c d_{2}}{8 \pi n m r}\right)\right) \\
& +O\left(\frac{K^{\epsilon}}{\sqrt{r}} \sum_{d \mid r^{2}} \sum_{n, m \leq K^{1+\epsilon}} \sum_{c \leq n m r / K^{2-\epsilon}} \frac{\left|S\left(n^{2}, m^{2} r^{2} / d_{2}^{2}, c\right)\right|}{n m \sqrt{c}}\right) .
\end{aligned}
$$

The error above is $O\left(r^{\frac{1}{2}} K^{\epsilon}\right)$ using Weil's bound on the Kloosterman sum. Now using Lemma 3.3 (with $b=m r / d_{2}$ and $d=d_{1} d_{2}^{2}$ ) we get that the main term of (3.23) is, up to an error of $O\left(K^{-10}\right)$,

$$
-K \frac{2 \sqrt{\pi}}{\sqrt{r}} \Im\left(e(-1 / 8) \sum_{d \mid r^{2}} \frac{1}{\sqrt{d_{1}}} \sum_{c, n, m \geq 1} \frac{\phi\left(c^{2}\right)}{c^{2}} \frac{1}{n m} W\left(\frac{n}{K}, \frac{m d_{1} d_{2}}{K}, \frac{K^{2} c^{2} d_{2}}{8 \pi n m r}\right)\right) .
$$

The point here is the 'completion of the square' which we saw in (3.7). This feature also appears in [5] and [7]. Note that the condition $r \leq K^{1-\epsilon}$ of Lemma 3.3 is the real limitation to the length of our mollifier.

We have by Lemma 3.5 that

$$
\begin{aligned}
W\left(\frac{n}{K}, \frac{m d_{1} d_{2}}{K}, \frac{K^{2} c^{2} d_{2}}{8 \pi n m r}\right)=\frac{1}{(2 \pi i)^{3}} \int_{(1)} & \int_{(1)} \int_{(1-\epsilon)} G(y) G(x) \frac{\zeta(1+2 y)}{y} \frac{\zeta(1+2 x)}{x} \\
& \times\left(\frac{K}{n}\right)^{y}\left(\frac{K}{m d_{1} d_{2}}\right)^{x}\left(\frac{8 \pi n m r}{K^{2} c^{2} d_{2}}\right)^{s} \widetilde{\hbar}_{x+y}(s) d s d y d x .
\end{aligned}
$$

Inserting this into (3.24) and exchanging orders of summation and integration freely by absolute convergence we get that (3.24) equals

$$
\begin{aligned}
& -K \frac{2 \sqrt{\pi}}{\sqrt{r}} \Im\left(e(-1 / 8) \frac{1}{(2 \pi i)^{3}} \int_{(1-\epsilon)} \int_{(1)} \int_{(1)}(8 \pi)^{s} G(y) G(x) \frac{\zeta(1+2 y)}{y} \frac{\zeta(1+2 x)}{x} \frac{K^{x+y}}{K^{2 s}}\right. \\
& \left.\quad \times \zeta(1+x-s) \zeta(1+y-s) \zeta(2 s) \zeta(2 s+1)^{-1} \widetilde{\hbar}_{x+y}(s) \sum_{d \mid r^{2}} \frac{r^{s}}{d_{1}^{x} d_{2}^{x+s} \sqrt{d_{1}}} d y d x d s\right),
\end{aligned}
$$

where we used that $\zeta(2 s) \zeta(2 s+1)^{-1}=\sum_{c \geq 1} \frac{\phi\left(c^{2}\right)}{c^{2 s+2}}$ for $\Re(s)>\frac{1}{2}$. Now we move the lines of integration $\Re(x)=\Re(y)=1$ to $\Re(x)=\Re(y)=\epsilon$, crossing simple poles at $y=s$ and $x=s$. On the new lines $\left|K^{x-s}\right| \ll K^{-1+\epsilon},\left|K^{y-s}\right| \ll K^{-1+\epsilon}$, so the contribution to (3.26) of the new integrals is $O\left(r^{\frac{1}{2}} K^{\epsilon}\right)$. Thus from the poles we get that (3.26) equals

$$
\begin{aligned}
-K \frac{2 \sqrt{\pi}}{\sqrt{r}} \Im\left(e(-1 / 8) \frac{1}{2 \pi i} \int_{(1-\epsilon)}(8 \pi)^{s} G(s)^{2}\right. & \frac{\zeta(1+2 s)^{2}}{s^{2}} \zeta(2 s) \zeta(2 s+1)^{-1} \widetilde{\hbar}_{2 s}(s) \\
& \left.\times \sum_{d \mid r^{2}} \frac{r^{s}}{d_{1}^{s} d_{2}^{2 s} \sqrt{d_{1}}} d s\right)+O\left(r^{\frac{1}{2}} K^{\epsilon}\right) .
\end{aligned}
$$


Using Lemma 3.5 the main term of (3.27) equals

$$
\begin{array}{r}
-K \frac{2 \sqrt{\pi}}{\sqrt{r}} \Im\left(e(-1 / 8) \frac{1}{2 \pi i} \int_{0}^{\infty} \int_{(1-\epsilon)} \frac{(8 \pi)^{s} G(s)^{2} \zeta(1+2 s) \zeta(2 s)}{s^{2}} \frac{h(\sqrt{u})}{\sqrt{2 \pi u}} \Gamma(s)\right. \\
\left.\times\left(\cos \left(\frac{\pi s}{2}\right)+i \sin \left(\frac{\pi s}{2}\right)\right) \sum_{d \mid r^{2}} \frac{1}{\sqrt{d_{1}}} \frac{r^{s}}{d^{s}} d s d u\right)
\end{array}
$$

$$
\begin{gathered}
=-K \frac{2 \sqrt{\pi}}{\sqrt{r}} \int_{0}^{\infty} \frac{h(\sqrt{u})}{\sqrt{2 \pi u}} d u \frac{1}{2 \pi i} \int_{(1-\epsilon)} \frac{(8 \pi)^{s} G(s)^{2} \zeta(1+2 s) \zeta(2 s)}{s^{2}} \Gamma(s) \sin \left(\frac{\pi s}{2}-\frac{\pi}{4}\right) \\
\times \sum_{d \mid r^{2}} \frac{1}{\sqrt{d_{1}}} \frac{r^{s}}{d^{s}} d s .
\end{gathered}
$$

We will see by the functional equation of $\zeta(s)$ that the integrand above is an even function of $s$. In fact (3.28) exactly cancels out with (3.21). This is another feature of the moment calculation.

We have $-2 \sqrt{\pi} \int_{0}^{\infty} \frac{h(\sqrt{u})}{\sqrt{2 \pi u}} d u=-2 \sqrt{2} \hat{h}(0)$, so to show that (3.28) and (3.21) cancel we need to show that $(8 \pi)^{s} \zeta(2 s) G(s) \Gamma(s) \sin \left(\frac{\pi s}{2}-\frac{\pi}{4}\right)=-\frac{1}{\sqrt{2}} \zeta(1-2 s) G(-s)$. By the functional equation of $\zeta(s)$ and the duplication formula of $\Gamma(s)$ we have

$$
\Gamma(s) \zeta(2 s)=\pi^{2 s-\frac{1}{2}} \Gamma\left(\frac{1}{2}-s\right) \zeta(1-2 s)=2^{-\frac{1}{2}-s} \pi^{2 s-1} \Gamma\left(\frac{1}{4}-\frac{s}{2}\right) \Gamma\left(\frac{3}{4}-\frac{s}{2}\right) \zeta(1-2 s) .
$$

By the definition of $G(s)$ given in Lemma 2.4 and the reflection formula of $\Gamma(s)$ we have

$$
G(s) \sin \left(\frac{\pi s}{2}-\frac{\pi}{4}\right)=\frac{\pi^{-\frac{3}{2} s} 2^{-s} \Gamma\left(\frac{s}{2}+\frac{3}{4}\right) \sin \left(\frac{\pi s}{2}-\frac{\pi}{4}\right)}{\Gamma\left(\frac{3}{4}\right)}=\frac{-\pi^{1-\frac{3}{2} s} 2^{-s}}{\Gamma\left(\frac{3}{4}\right) \Gamma\left(\frac{1}{4}-\frac{s}{2}\right)} .
$$

Combining (3.29) and (3.30) gives the required formula for $(8 \pi)^{s} \zeta(2 s) G(s) \Gamma(s) \sin \left(\frac{\pi s}{2}\right.$ $\left.-\frac{\pi}{4}\right)$.

\section{Optimizing the MOLLIFIER}

In the previous sections we found the first and second moments of $L\left(\frac{1}{2}, \operatorname{sym}^{2} f\right)$ twisted by the Fourier coefficient $a_{f}\left(r^{2}\right)$. These immediately lead to the moments of $L\left(\frac{1}{2}, \operatorname{sym}^{2} f\right)$ times the mollifier $M(f)=\sum_{r} a_{f}\left(r^{2}\right) x_{r} r^{-\frac{1}{2}}$. Here $x_{r}$ are coefficients to be determined with $x_{r}=0$ for $r \leq 0$ or $r>M$, where $M=K^{a}$ for some constant $a>0$ also to be determined. Also suppose that $x_{r} \ll M^{\epsilon}$ as this will be the case once choices are made. In this chapter we choose a mollifier that maximizes the proportion of non-vanishing of $L\left(\frac{1}{2}, \operatorname{sym}^{2} f\right)$ given by (1.19). We shall see that this boils down to a problem of minimizing a quadratic form in the $x_{r}$ with a linear constraint. By Theorem 2.1 we have the first mollified moment, a linear form in $x_{r}:$

$$
\begin{aligned}
\mathcal{M}_{1} & =\sum_{k \equiv 0 \bmod 2} h\left(\frac{k-1}{K}\right) \sum_{f \in H_{k}}^{h} L\left(\frac{1}{2}, \operatorname{sym}^{2} f\right) \sum_{r} \frac{x_{r}}{\sqrt{r}} a_{f}\left(r^{2}\right) \\
& =\frac{K}{2} \sum_{r} \frac{x_{r}}{r} \int_{0}^{\infty} h(u)(\log (u K / r)+C) d u+O\left(M K^{\epsilon}\right) .
\end{aligned}
$$


Using the Hecke relation (3.13) we have the second mollified moment, a quadratic form in $x_{r}$ :

$$
\begin{aligned}
\mathcal{M}_{2} & =\sum_{k \equiv 0 \bmod 2} h\left(\frac{k-1}{K}\right) \sum_{f \in H_{k}}^{h} L\left(\frac{1}{2}, \operatorname{sym}^{2} f\right)^{2}\left(\sum_{r} \frac{x_{r}}{\sqrt{r}} a_{f}\left(r^{2}\right)\right)^{2} \\
& =\sum_{k \equiv 0 \bmod 2} h\left(\frac{k-1}{K}\right) \sum_{f \in H_{k}}^{h} L\left(\frac{1}{2}, \operatorname{sym}^{2} f\right)^{2} \sum_{\alpha \geq 1} \sum_{e \mid \alpha^{2}} \sum_{\substack{r_{1}, r_{2} \\
\left(r_{1}, r_{2}\right)=1}} \frac{x_{\alpha r_{1}} x_{\alpha r_{2}}}{\alpha \sqrt{r_{1} r_{2}}} a_{f}\left(\frac{r_{1}^{2} r_{2}^{2} \alpha^{4}}{e^{2}}\right) .
\end{aligned}
$$

Above $\frac{r_{1} r_{2} \alpha^{2}}{e} \leq K^{1-\epsilon}$ if $M \leq K^{\frac{1}{2}-\epsilon}$. Thus under this condition we have by Theorem 3.1 .

$$
\mathcal{M}_{2}=\frac{K}{2} \sum_{\alpha} \sum_{e \mid \alpha^{2}} \sum_{\substack{r_{1}, r_{2} \\\left(r_{1}, r_{2}\right)=1}} x_{\alpha r_{1}} x_{\alpha r_{2}} \frac{\sqrt{e}}{\alpha^{2} r_{1} r_{2}} S\left(\frac{r_{1} r_{2} \alpha^{2}}{e}\right)+O\left(M^{2} K^{\epsilon}\right)
$$

where

$$
\begin{gathered}
S(r)=\sum_{d \mid r^{2}} \frac{1}{\sqrt{d_{1}}} \int_{0}^{\infty} h(u)\left(\frac{1}{2} \log ^{2} \frac{u K}{d_{1} d_{2}} \log \frac{u K d_{2}}{r}-\frac{1}{6} \log ^{3} \frac{u K}{d_{1} d_{2}}+2 P_{2}\left(\log \frac{u K}{d_{1} d_{2}}\right)\right. \\
\left.+2 \log \frac{u K d_{2}}{r} P_{1}\left(\log \frac{u K}{d_{1} d_{2}}\right)\right) d u
\end{gathered}
$$

and $P_{i}$ are degree $i$ polynomials as in Theorem 3.1. We see from this that the longest length of mollifier we are able to take is $M=K^{\frac{1}{2}-\epsilon}$ and hence we make the restriction $a<\frac{1}{2}$. Define a multiplicative function

$$
\tau(n)=\sum_{d \mid n^{2}} \frac{1}{\sqrt{d}_{1}}=\sum_{f \mid n} \frac{\mu^{2}(f)}{\sqrt{f}} d(n / f),
$$

where in the second sum $d$ is the divisor function. Note that $\tau(p)=2+p^{-\frac{1}{2}}$, so that $\tau$ behaves roughly like the divisor function. Since $S(r)$ is roughly of size $(\log K)^{3}$ for $r \leq K^{1-\epsilon}$, we expect $\mathcal{M}_{2}$ to be roughly of size

$$
K(\log K)^{3} \sum_{\alpha} \sum_{e \mid \alpha^{2}} \sum_{\substack{r_{1}, r_{2} \\\left(r_{1}, r_{2}\right)=1}} \frac{\sqrt{e}}{\alpha^{2} r_{1} r_{2}} \tau\left(\frac{r_{1} r_{2} \alpha^{2}}{e}\right) x_{\alpha r_{1}} x_{\alpha r_{2}}
$$

We will make a choice of $x_{r}$ based on this quadratic form; one that maximizes the ratio of $\mathcal{M}_{1}^{2}$ to (4.6), under the assumption that $x_{r}$ is supported on square-free $r$. The form of our mollifier and this last assumption are reasonable from the Euler product (1.3) and the expectation that the mollifier will mimic a multiple of the inverse of $L\left(\frac{1}{2}, \operatorname{sym}^{2} f\right)$. For this choice we will find that $\mathcal{M}_{1} \asymp K(\log K)^{3}$ and $\mathcal{M}_{2} \asymp K(\log K)^{6}$, and we'll get that

$$
\frac{\mathcal{M}_{1}^{2}}{\mathcal{M}_{2}} \sim\left(1-\frac{1}{(1+a)^{3}}\right) \sum_{k \equiv 0 \bmod 2} h\left(\frac{k}{K}\right) \sum_{f \in H_{k}}^{h} 1 .
$$

Thus together with observation (1.19) the main theorem will follow. 
4.1. Change of variables. We simplify the problem by diagonalizing the quadratic form (4.6). We assume that $x_{r}=0$ if $r$ is not square-free. We have

$$
\begin{aligned}
& \sum_{\alpha} \sum_{e \mid \alpha^{2}} \sum_{\substack{r_{1}, r_{2} \\
\left(r_{1}, r_{2}\right)=1}} \frac{\sqrt{e}}{\alpha^{2} r_{1} r_{2}} \tau\left(\frac{r_{1} r_{2} \alpha^{2}}{e}\right) x_{\alpha r_{1}} x_{\alpha r_{2}} \\
& =\sum_{\alpha} \sum_{e \mid \alpha^{2}} \sum_{\substack{r_{1}, r_{2} \\
\left(r_{1}, r_{2}\right)=1}} \frac{\sqrt{e}}{\alpha^{2} r_{1} r_{2}} \tau\left(r_{1}\right) \tau\left(r_{2}\right) \tau\left(\alpha^{2} / e\right) x_{\alpha r_{1}} x_{\alpha r_{2}} \\
& =\sum_{\alpha, \beta \geq 1} \sum_{e \mid \alpha^{2}} \sum_{r_{1}, r_{2}} \frac{\mu(\beta) \sqrt{e}}{\beta^{2} \alpha^{2} r_{1} r_{2}} \tau(\beta)^{2} \tau\left(r_{1}\right) \tau\left(r_{2}\right) \tau\left(\alpha^{2} / e\right) x_{\alpha \beta r_{1}} x_{\alpha \beta r_{2}},
\end{aligned}
$$

where in the last line we used the Mobius function to detect the condition $\left(r_{1}, r_{2}\right)=$ 1. Define a change of variables

$$
y_{j}=\sum_{r} \frac{\tau(r)}{r} x_{j r} .
$$

Thus $y_{j}$ is supported on positive square-free integers $j$ less than or equal to $M$. This change of variables is invertible:

$$
x_{r}=\sum_{n} \frac{x_{n r} \tau(n)}{n} \sum_{d \mid n} \mu(d)=\sum_{d} \frac{\tau(d) \mu(d)}{d} \sum_{n} \frac{x_{n d r} \tau(n)}{n}=\sum_{d} \frac{y_{d r} \tau(d) \mu(d)}{d},
$$

where we used the Mobius function to detect $n=1$ in the first sum. Also define coefficients for positive square-free $j$ (and otherwise set equal to zero),

$$
v_{j}=\frac{1}{j^{2}} \sum_{\alpha \beta=j} \mu(\beta) \tau(\beta)^{2} \sum_{e \mid \alpha^{2}} \sqrt{e} \tau\left(\frac{\alpha^{2}}{e}\right)=\frac{1}{j} \prod_{p \mid j}\left(1+\frac{2}{p^{\frac{1}{2}}}-\frac{2}{p^{\frac{3}{2}}}-\frac{1}{p^{2}}\right) .
$$

Then we have that (4.8) equals

$$
\sum_{j} v_{j} y_{j}^{2}
$$

and (4.6) equals $K \log ^{3} K \sum_{j} v_{j} y_{j}^{2}$. This is the diagonal quadratic form that we will work with. For this change of variables, the linear form $\mathcal{M}_{1}$ equals

$$
\mathcal{M}_{1}=\frac{\hat{h}(0) K}{2} \sum_{j} u_{j} y_{j}+O\left(M^{2} K^{\epsilon}\right)
$$

where the coefficients are

$$
u_{j}=\frac{1}{\hat{h}(0)} \frac{1}{j} \sum_{n r=j} \mu(n) \tau(n) \int_{0}^{\infty} h(u)(\log (u K / r)+C) d u .
$$

For square-free $j$ we have

$$
u_{j}=\frac{1}{j} \sum_{n \mid j} \mu(n) \tau(n) \log (K n / j)+O\left(j^{-1} \mu_{*}(j)\right),
$$

where we define for positive square-free $j$ (and otherwise set equal to zero),

$$
\mu_{*}(j)=\sum_{n \mid j} \mu(n) \tau(n)=\prod_{p \mid j}\left(-1-\frac{1}{\sqrt{p}}\right) .
$$


As the notation suggests, $\mu_{*}$ behaves roughly like $\mu$. Now,

$$
\begin{aligned}
& \sum_{n \mid j} \mu(n) \tau(n) \log (K n / j)=\mu_{*}(j) \log K-\sum_{n \mid j} \mu(n) \tau(n) \sum_{p \mid \frac{j}{n}} \log p \\
& =\mu_{*}(j) \log K-\sum_{p \mid j} \log p \sum_{n \mid \frac{j}{p}} \mu(n) \tau(n)=\mu_{*}(j) \log K-\mu_{*}(j) \sum_{p \mid j} \frac{\log p}{\mu_{*}(p)} \\
& =\mu_{*}(j) \log K-\mu_{*}(j) \sum_{p \mid j} \log p\left(-1+O\left(p^{-\frac{1}{2}}\right)\right) \\
& =\mu_{*}(j) \log (K j)+O\left(\prod_{p \mid j}\left(1+p^{-\frac{1}{2}+\epsilon}\right)\right)
\end{aligned}
$$

Thus

$$
u_{j}=\frac{\mu_{*}(j)}{j} \log (K j)+O\left(j^{-1} \prod_{p \mid j}\left(1+p^{-\frac{1}{2}+\epsilon}\right)\right) .
$$

With this change of variables we can easily determine the optimum choice for $y_{j}$. By the Cauchy-Schwarz inequality we have,

$$
\left(\sum_{j} u_{j} y_{j}\right)^{2}=\left(\sum_{j} \frac{u_{j}}{\sqrt{v_{j}}} \sqrt{v_{j}} y_{j}\right)^{2} \leq\left(\sum_{j} v_{j} y_{j}^{2}\right)\left(\sum_{j} \frac{u_{j}^{2}}{v_{j}}\right) .
$$

Thus $\left(\sum_{j} u_{j} y_{j}\right)^{2} / \sum_{j} v_{j} y_{j}^{2}$ obtains its upper bound when

$$
y_{j}=\frac{u_{j}}{v_{j}} .
$$

Set $y_{j}$ to this value henceforth and note that $y_{j} \ll \log K$.

4.2. Evaluating $\mathcal{M}_{1}$. For the choice made in (4.20) we have

$$
\begin{aligned}
\mathcal{M}_{1} & =\frac{\hat{h}(0) K}{2} \sum_{j \leq M} \frac{u_{j}^{2}}{v_{j}} \\
& =\frac{\hat{h}(0) K}{2} \sum_{j \leq M} \frac{\mu_{*}(j)^{2}}{j^{2} v_{j}} \log ^{2}(K j)+O\left(K \log K \sum_{j \leq M} j^{-1} \prod_{p \mid j}\left(1+p^{-\frac{1}{2}+\epsilon}\right)\right) .
\end{aligned}
$$

The error is $O\left(K \log ^{2} K\right)$. We have that

$$
\frac{\mu_{*}(p)^{2}}{p^{2} v_{p}}=\frac{1+2 p^{-\frac{1}{2}}+p^{-1}}{p\left(1+2 p^{-\frac{1}{2}}-2 p^{-\frac{3}{2}}-p^{-2}\right)}=\frac{1}{p\left(1-p^{-1}\right)}=\frac{1}{\phi(p)},
$$

after a nice simplification. Now it is well known that

$$
\sum_{j \leq x} \frac{\mu_{*}(j)^{2}}{j^{2} v_{j}}=\sum_{j \leq x} \frac{\mu(j)^{2}}{\phi(j)}=\log x+O(1) .
$$

Indeed, we have that $\sum_{j \geq 1} \frac{\mu(j)^{2}}{\phi(j)} \frac{1}{j^{s}}=\prod_{p}\left(1+p^{-(1+s)}+p^{-(2+s)}+\ldots\right)=\zeta(s+1) F(s)$, where $F(s)$ is analytic and absolutely convergent for $\Re(s)>-\frac{1}{2}$ and $F(0)=1$. 
By partial summation we get

$$
\begin{aligned}
& \mathcal{M}_{1}= \frac{\hat{h}(0) K}{2} \sum_{j \leq M} \frac{\mu_{*}(j)^{2}}{j^{2} v_{j}} \log ^{2}(K j)+O\left(K \log ^{2} K\right) \\
&= \frac{\hat{h}(0) K}{2} \log ^{2}(K M) \log M-\frac{\hat{h}(0) K}{2} \sum_{j \leq M}\left(\log ^{2}(K(j+1))-\log ^{2}(K j)\right) \log j \\
& \quad+O\left(K \log ^{2} K\right) \\
&(4.24) \sim \hat{h}(0) K \log ^{3} K \frac{a\left(3+3 a+a^{2}\right)}{6} .
\end{aligned}
$$

4.3. Evaluating $\mathcal{M}_{2}$. Expanding the logarithms in the definition of $S\left(r_{1} r_{2} \alpha^{2} / e\right)$, we see that $\mathcal{M}_{2}$ is a sum of terms proportional to

$$
\begin{gathered}
K(\log K)^{i_{0}} \sum_{\alpha} \sum_{e \mid \alpha^{2}} \sum_{\substack{r_{1}, r_{2} \\
\left(r_{1}, r_{2}\right)=1}} \frac{\sqrt{e}}{r_{1} r_{2}} \frac{\log \left(\alpha^{2} / e\right)^{i_{1}}}{\alpha^{2}} \sum_{d \mid r_{1}^{2} r_{2}^{2} \alpha^{4} / e^{2}} \frac{\left(\log d_{1}\right)^{i_{2}}\left(\log d_{2}\right)^{i_{3}}}{\sqrt{d_{1}}} \\
\left(\log r_{1} r_{2}\right)^{i_{4}}\left(\int_{0}^{\infty} h(u)(\log u)^{i_{5}} d u\right) x_{\alpha r_{1}} x_{\alpha r_{2}},
\end{gathered}
$$

where $i_{0}, \ldots, i_{5}$ are non-negative integers with $i_{0}+\ldots+i_{5}=3, i_{1} \leq 1$ and $i_{4} \leq 1$. We show in this section that (4.25) is of size $K(\log K)^{6}$ when $i_{0}+i_{3}+i_{4}=3$. In the next section we will see that it is bounded by $K(\log K)^{5}$ otherwise. Thus in this section we want to evaluate the quadratic form,

$$
\frac{K \hat{h}(0)}{2} \sum_{\alpha} \sum_{e \mid \alpha^{2}} \sum_{\substack{r_{1}, r_{2} \\\left(r_{1}, r_{2}\right)=1}} \frac{\sqrt{e}}{\alpha^{2} r_{1} r_{2}} \sum_{d \mid r_{1}^{2} r_{2}^{2} \alpha^{4} / e^{2}}\left(\frac{1}{2} \log ^{2} \frac{K}{d_{2}} \log \frac{K d_{2}}{r_{1} r_{2}}-\frac{1}{6} \log ^{3} \frac{K}{d_{2}}\right) x_{\alpha r_{1}} x_{\alpha r_{2}},
$$

for the choice made in (4.20). We have

$$
\begin{array}{r}
\frac{1}{2} \log ^{2} \frac{K}{d_{2}} \log \frac{K d_{2}}{r_{1} r_{2}}-\frac{1}{6} \log ^{3} \frac{K}{d_{2}}=\frac{1}{3} \log ^{3} K-\frac{1}{2} \log ^{2} K \log r_{1} r_{2}+\log K \log r_{1} r_{2} \log d_{2} \\
-\log K \log ^{2} d_{2}+\frac{2}{3} \log ^{3} d_{2}-\frac{1}{2} \log r_{1} r_{2} \log ^{2} d_{2},
\end{array}
$$

and we evaluate the contribution of each of these terms one by one.

- The contribution of the $\log ^{3} K$ term of (4.27) to (4.26) is

$$
\frac{K \hat{h}(0)}{6} \sum_{j} v_{j} y_{j}^{2}=\frac{K \hat{h}(0)}{6} \sum_{j \leq M} \frac{u_{j}^{2}}{v_{j}} \sim \hat{h}(0) K \log ^{6} K \frac{a\left(3+3 a+a^{2}\right)}{18},
$$

by the same calculations as for the evaluation of $\mathcal{M}_{1}$.

- The contribution of the $\log ^{2} K \log r_{1} r_{2}$ term of (4.27) to (4.26) is

$$
\begin{aligned}
& \frac{-\hat{h}(0) K \log ^{2} K}{4} \sum_{\alpha} \sum_{e \mid \alpha^{2}} \sum_{\substack{r_{1}, r_{2} \\
\left(r_{1}, r_{2}\right)=1}} \frac{\sqrt{e}}{\alpha^{2} r_{1} r_{2}} \tau\left(\frac{r_{1} r_{2} \alpha^{2}}{e}\right) \log \left(r_{1} r_{2}\right) x_{\alpha r_{1}} x_{\alpha r_{2}} \\
& =\frac{-\hat{h}(0) K \log ^{2} K}{2} \sum_{\alpha} \sum_{e \mid \alpha^{2}} \sum_{\substack{r_{1}, r_{2} \\
\left(r_{1}, r_{2}\right)=1}} \frac{\sqrt{e}}{\alpha^{2} r_{1} r_{2}} \tau\left(\frac{r_{1} r_{2} \alpha^{2}}{e}\right) \log \left(r_{1}\right) x_{\alpha r_{1}} x_{\alpha r_{2}},
\end{aligned}
$$


RIZWANUR KHAN

by symmetry. Using the Mobius function to detect $\left(r_{1}, r_{2}\right)=1$ this equals

$$
\begin{aligned}
& \frac{-\hat{h}(0) K \log ^{2} K}{2} \sum_{\alpha, \beta} \sum_{e \mid \alpha^{2}} \frac{\mu(\beta) \tau(\beta)^{2} \sqrt{e} \tau\left(\alpha^{2} / e\right)}{\alpha^{2} \beta^{2}} \sum_{r_{1}, r_{2}} \frac{\tau\left(r_{1}\right) \tau\left(r_{2}\right) \log \left(\beta r_{1}\right)}{r_{1} r_{2}} x_{\alpha \beta r_{1}} x_{\alpha \beta r_{2}} \\
& =\frac{-\hat{h}(0) K \log ^{2} K}{2} \sum_{j} v_{j} y_{j} \sum_{r_{1}} \frac{\tau\left(r_{1}\right) \log \left(r_{1}\right)}{r_{1}} x_{j r_{1}} \\
& +\frac{-\hat{h}(0) K \log ^{2} K}{2} \sum_{\alpha, \beta}\left(\frac{1}{(\alpha \beta)^{2}} \mu(\beta) \tau(\beta)^{2} \log \beta \sum_{e \mid \alpha^{2}} \sqrt{e} \tau\left(\alpha^{2} / e\right)\right) y_{\alpha \beta}^{2},
\end{aligned}
$$

in terms of the new variables $y_{j}$. Using that $\alpha^{-2} \sum_{e \mid \alpha^{2}} \sqrt{e} \tau\left(\alpha^{2} / e\right) \ll \alpha^{-1} \prod(1+$ $p^{-\frac{1}{2}+\epsilon}$ ) and the bound $y_{j} \ll \log K$, we have that the second line of (4.30) is $O\left(\log ^{5} K\right)$.

Let $\Lambda_{j}(n)=\sum_{d \mid n} \mu(d)\left(\log \frac{n}{d}\right)^{j}$ for $j \geq 0$ be the generalized von Mangoldt function. Thus $\Lambda_{0}(1)=1$ and $\Lambda_{0}(n)=0$ for $n \geq 2$, and $\Lambda_{1}(n)$ is the usual von Mangoldt function $\Lambda(n)$ supported on prime powers. The function $\Lambda_{j}(n)$ is supported on integers $n$ having at most $j$ distinct prime factors and satisfies $\Lambda_{j}(n) \ll_{j}(\log n)^{j}, \sum_{n \leq x} \Lambda_{j}(n) / n \ll_{j}(\log x)^{j}$ and $(\log n)^{j}=\sum_{d \mid n} \Lambda_{j}(d)$. We also have $\Lambda_{j}(n m)=\sum_{0 \leq i \leq j}\left(\begin{array}{l}j \\ i\end{array}\right) \Lambda_{i}(n) \Lambda_{j-i}(m)$ for coprime integers $n$ and $m$. Writing $\log r_{1}=\sum_{b \mid r_{1}} \Lambda(b)$, the first term of (4.30) is

$$
\begin{aligned}
& \frac{-\hat{h}(0) K \log ^{2} K}{2} \sum_{j} v_{j} \sum_{b} \frac{\Lambda(b) \tau(b)}{b} y_{b j} y_{j} \\
& =\frac{-\hat{h}(0) K \log ^{2} K}{2} \sum_{j \leq M} \frac{\mu_{*}(j)^{2}}{j^{2} v_{j}} \sum_{\substack{b \leq M / j \\
(b, j)=1}} \frac{\Lambda(b) \tau(b)}{b} \frac{\mu_{*}(b)}{b v_{b}} \log (K j) \log (K j b) \\
& \quad+O\left(K \log ^{5} K\right),
\end{aligned}
$$

on substituting the value $u_{j} / v_{j}$ of $y_{j}$ and using (4.17). As the sum above is restricted to prime values of $b$, we can remove the condition $(b, j)=1$ since otherwise $b \mid j$ and the contribution of such terms is $\ll K \log ^{5} K$. The above sum can be evaluated by partial summation. Recall that $\frac{\mu_{*}(j)^{2}}{j^{2} v_{j}}=\frac{\mu(j)^{2}}{\phi(j)}$ behaves like $\frac{1}{j}$ on average, and for prime $b, \tau(b)=2+b^{-\frac{1}{2}}, \mu_{*}(b)=-1-b^{-\frac{1}{2}}$ and $b v_{b}=1+O\left(b^{-\frac{1}{2}}\right)$. Using the prime number theorem we get that (4.31) is

$$
\sim \frac{-\hat{h}(0) K \log ^{2} K}{2} \sum_{j \leq M} \frac{\mu_{*}(j)^{2}}{j^{2} v_{j}}\left(-2 \log (M / j) \log ^{2}(K j)-\log ^{2}(M / j) \log (K j)\right)
$$

$$
\sim \hat{h}(0) K \log ^{6} K \frac{a^{2}(2+a)^{2}}{8},
$$

by partial summation. 
- The contribution of the $\log K \log r_{1} r_{2} \log d_{2}$ term of (4.27) to (4.26) is

$$
\hat{h}(0) K \log K \sum_{\alpha} \sum_{e \mid \alpha^{2}} \sum_{\substack{r_{1}, r_{2} \\\left(r_{1}, r_{2}\right)=1}} \frac{\sqrt{e}}{\alpha^{2} r_{1} r_{2}}\left(\sum_{d \mid r_{1}^{2} r_{2}^{2} \alpha^{4} / e^{2}} \frac{\log d_{2}}{\sqrt{d_{1}}}\right) \log r_{1} x_{\alpha r_{1}} x_{\alpha r_{2}} .
$$

Note that

$$
\sum_{d \mid n^{2}} \frac{\log ^{i} d_{1} \log ^{j} d_{2}}{\sqrt{d_{1}}}=\sum_{f \mid n} \frac{\mu(f)^{2} \log ^{i} f}{\sqrt{f}} \sum_{e \mid \frac{n}{f}} \log ^{j} e=\sum_{a \mid n} \sum_{b \mid \frac{n}{a}} \frac{\mu(a)^{2} \Lambda_{i}(a) \Lambda_{j}(b)}{\sqrt{a}} \tau\left(\frac{n}{a b}\right) .
$$

Using this we get

$$
\begin{gathered}
\sum_{d \mid r_{1}^{2} r_{2}^{2} \alpha^{4} / e^{2}} \frac{\log d_{2}}{\sqrt{d_{1}}}=\left(\sum_{c \mid r_{1}} \Lambda(c) \tau\left(r_{1} / c\right)\right) \tau\left(r_{2}\right) \tau\left(\alpha^{2} / e\right)+\tau\left(r_{1}\right)\left(\sum_{c \mid r_{2}} \Lambda(c) \tau\left(r_{2} / c\right)\right) \tau\left(\alpha^{2} / e\right) \\
+\tau\left(r_{1}\right) \tau\left(r_{2}\right)\left(\sum_{c \mid \alpha^{2} / e} \Lambda(c) \tau\left(\alpha^{2} / e c\right)\right) .
\end{gathered}
$$

Thus (4.33) is

$$
\begin{aligned}
& \hat{h}(0) K \log K \\
& \times\left(\sum_{\alpha, \beta} \sum_{e \mid \alpha^{2}} \sum_{c} \frac{\sqrt{e} \tau(\beta)^{2} \mu(\beta) \tau\left(\alpha^{2} / e\right)}{\alpha^{2} \beta^{2}} \frac{\Lambda(c)}{c} \sum_{\substack{r_{1}, r_{2} \\
c \nmid r_{2}}} \frac{x_{\alpha \beta c r_{1}} x_{\alpha \beta r_{2}} \tau\left(r_{1}\right) \tau\left(r_{2}\right) \log \left(c \beta r_{1}\right)}{r_{1} r_{2}}\right. \\
& +\sum_{\alpha, \beta} \sum_{e \mid \alpha^{2}} \sum_{c} \frac{\sqrt{e} \tau(\beta)^{2} \mu(\beta) \tau\left(\alpha^{2} / e\right)}{\alpha^{2} \beta^{2}} \frac{\Lambda(c)}{c} \sum_{\substack{r_{1}, r_{2} \\
c \nmid r_{1}}} \frac{x_{\alpha \beta r_{1}} x_{\alpha \beta c r_{2}} \tau\left(r_{1}\right) \tau\left(r_{2}\right) \log \left(\beta r_{1}\right)}{r_{1} r_{2}} \\
& \left.+\sum_{\alpha, \beta} \sum_{e \mid \alpha^{2}}\left(\frac{\sqrt{e} \tau(\beta)^{2} \mu(\beta)}{\alpha^{2} \beta^{2}} \sum_{c \mid \alpha^{2} / e} \Lambda(c) \tau\left(\alpha^{2} / e c\right)\right) \sum_{r_{1}, r_{2}} \frac{x_{\alpha \beta r_{1}} x_{\alpha \beta r_{2}} \tau\left(r_{1}\right) \tau\left(r_{2}\right) \log \left(\beta r_{1}\right)}{r_{1} r_{2}}\right) .
\end{aligned}
$$

In terms of the new variables $y_{j}$, the first two lines of (4.36) contribute

$$
\begin{aligned}
\hat{h}(0) K \log K \sum_{j} v_{j}\left(\sum_{c} \frac{\Lambda(c) \log c}{c} y_{c j} y_{j}+\sum_{c, b} \frac{\Lambda(c)}{c} \frac{\Lambda(b) \tau(b)}{b} y_{c b j} y_{j}\right. \\
\left.+\sum_{c, b} \frac{\Lambda(c)}{c} \frac{\Lambda(b) \tau(b)}{b} y_{c j} y_{b j}\right)+O\left(K \log ^{5} K\right)
\end{aligned}
$$

$$
\begin{aligned}
\sim \hat{h}(0) K \log K & \sum_{j \leq M} \frac{\mu_{*}(j)^{2}}{j^{2} v_{j}}\left(\sum_{c \leq M / j} \frac{\Lambda^{2}(c)}{c} \frac{\mu_{*}(c)}{c v_{c}} \log (K j c) \log (K j)\right. \\
& +\sum_{b c \leq M / j} \frac{\Lambda(c) \Lambda(b) \tau(b)}{c b} \frac{\mu_{*}(c b)}{c b v_{c b}} \log (K j c b) \log (K j) \\
& \left.+\sum_{\substack{c \leq M / j \\
b \leq M / j}} \frac{\Lambda(c) \Lambda(b) \tau(b)}{c b} \frac{\mu_{*}(c) \mu_{*}(b)}{c v_{c} b v_{b}} \log (K j c) \log (K j b)\right) .
\end{aligned}
$$


By the prime number theorem this is

$$
\begin{aligned}
\sim \hat{h}(0) K \log K \sum_{j \leq M} \frac{\mu_{*}(j)^{2}}{j^{2} v_{j}}(- & \frac{1}{2} \log ^{2}(M / j) \log ^{2}(K j)-\frac{1}{3} \log ^{3}(M / j) \log (K j) \\
+ & 2\left(\log (M / j) \log (K j)+\frac{1}{2} \log ^{2}(M / j)\right)^{2} \\
+ & \left.\log ^{2}(M / j) \log ^{2}(K j)+\frac{2}{3} \log ^{3}(M / j) \log (K j)\right) .
\end{aligned}
$$

We will evaluate (4.38) by partial summation after combining it with (4.43), the contribution of the $\log K \log ^{2} d_{2}$ term. As for the third line of (4.36), we have

$$
\sum_{e \mid \alpha^{2}} \frac{\sqrt{e}}{\alpha^{2}} \sum_{c \mid \alpha^{2} / e} \Lambda(c) \tau\left(\alpha^{2} / e c\right)=\frac{1}{\alpha} \sum_{c \mid \alpha^{2}} \frac{\Lambda(c)}{\sqrt{c}} \sum_{e \mid \alpha^{2} / c} \frac{\tau(e)}{\sqrt{e}} \ll \frac{1}{\alpha} \prod_{p \mid \alpha}\left(1+p^{-\frac{1}{2}+\epsilon}\right) .
$$

So the contribution of the third line is $O\left(K \log ^{5} K\right)$.

- The contribution of the $\log K \log ^{2} d_{2}$ term of (4.27) to (4.26) is

$$
\frac{-\hat{h}(0) K \log K}{2} \sum_{\alpha} \sum_{e \mid \alpha^{2}} \sum_{\substack{r_{1}, r_{2} \\\left(r_{1}, r_{2}\right)=1}} \frac{\sqrt{e}}{\alpha^{2} r_{1} r_{2}}\left(\sum_{d \mid r_{1}^{2} r_{2}^{2} \alpha^{4} / e^{2}} \frac{\log ^{2} d_{2}}{\sqrt{d_{1}}}\right) x_{\alpha r_{1}} x_{\alpha r_{2}} .
$$

We have

$$
\begin{aligned}
& \sum_{d \mid r_{1}^{2} r_{2}^{2} \alpha^{4} / e^{2}} \frac{\log ^{2} d_{2}}{\sqrt{d_{1}}} \\
& =\sum_{\substack{i+j+k=2 \\
i, j, k \geq 0}} a_{i j k}\left(\sum_{c \mid r_{1}} \Lambda_{i}(c) \tau\left(r_{1} / c\right)\right)\left(\sum_{c \mid r_{2}} \Lambda_{j}(c) \tau\left(r_{2} / c\right)\right)\left(\sum_{c \mid \alpha^{2} / e} \Lambda_{k}(c) \tau\left(\alpha^{2} / e c\right)\right),
\end{aligned}
$$

for some coefficients $a_{i j k}$, with $a_{110}=2$. Only the term with $i=j=1$ and $k=0$ of this expansion is significant. Showing that the contribution to (4.40) of any term with $k \geq 1$ is $O\left(K \log ^{5} K\right)$ is very similar to how we showed that third term of (4.36) is small. This leaves us to consider the contribution of the term with $i=2$ and $j=k=0$. This is less than

$$
\begin{aligned}
& K \log K \sum_{j} v_{j} \sum_{c} \frac{\Lambda_{2}(c)}{c} y_{c j} y_{j}+O\left(K \log ^{5} K\right) \\
& \ll K \log K \sum_{j \leq M} \frac{\mu_{*}(j)^{2}}{j v_{j}} \sum_{c \leq M / j} \frac{\Lambda_{2}(c)}{c} \frac{\mu_{*}(c)}{c v_{c}} \log (K j c) \log (K j)+O\left(K \log ^{5} K\right) \\
& \ll K \log ^{5} K,
\end{aligned}
$$

where the last step follows by the estimate $\sum_{c \leq x} \frac{\Lambda_{2}(c) \mu_{*}(c)}{c^{2} v_{c}} \ll \log x$. To see this estimate, note that for prime values of $c$ the sum is $\sum_{p \leq x} \frac{-(\log p)^{2}}{p}\left(1+O\left(\frac{1}{\sqrt{p}}\right)\right)=$ $-\frac{1}{2}(\log x)^{2}+O(\log x)$ by the prime number theorem, and for $c$ a product of two distinct primes the sum is $\sum_{\substack{p q \leq x \\ p \neq q}} \frac{2 \log p \log q}{p q}\left(1+O\left(\frac{1}{\sqrt{p}}+\frac{1}{\sqrt{q}}\right)\right)=\frac{1}{2}(\log x)^{2}+O(\log x)$. 
Thus (4.40) is

$$
\begin{aligned}
& \sim \frac{-\hat{h}(0) K \log K}{2} \sum_{j} 2 v_{j}\left(\sum_{c} \frac{\Lambda(c)}{c} y_{j c}\right)^{2} \\
& \sim-\hat{h}(0) K \log K \sum_{j \leq M} \frac{\mu_{*}(j)^{2}}{j v_{j}}\left(\sum_{c \leq M / j} \frac{\Lambda(c)}{c} \frac{\mu_{*}(c)}{c v_{c}} \log (K j c)\right)^{2} \\
& \sim-\hat{h}(0) K \log K \sum_{j \leq M} \frac{\mu_{*}(j)^{2}}{j v_{j}}\left(\log (M / j) \log (K j)+\frac{1}{2} \log ^{2}(M / j)\right)^{2} .
\end{aligned}
$$

We combine this with the contribution of the previous calculation. The sum of (4.43) and (4.38) is

$$
\hat{h}(0) K \log K \sum_{j \leq M} \frac{\mu_{*}(j)^{2}}{j v_{j}}\left(\frac{3}{2} \log ^{2}(M / j) \log ^{2}(K j)+\frac{4}{3} \log ^{3}(M / j) \log (K j)+\frac{1}{4} \log ^{4}(M / j)\right)
$$

$$
\sim \hat{h}(0) K \log ^{6} K \frac{a^{3}\left(6+7 a+2 a^{2}\right)}{12} .
$$

- The contribution of the $\log ^{3} d_{2}$ term of (4.27) to (4.26) is

$$
\frac{\hat{h}(0) K}{3} \sum_{\alpha} \sum_{e \mid \alpha^{2}} \sum_{\substack{r_{1}, r_{2} \\\left(r_{1}, r_{2}\right)=1}} \frac{\sqrt{e}}{\alpha^{2} r_{1} r_{2}}\left(\sum_{d \mid r_{1}^{2} r_{2}^{2} \alpha^{4} / e^{2}} \frac{\log ^{3} d_{2}}{\sqrt{d_{1}}}\right) x_{\alpha r_{1}} x_{\alpha r_{2}} .
$$

We have

$$
\begin{aligned}
& \sum_{d \mid r_{1}^{2} r_{2}^{2} \alpha^{4} / e^{2}} \frac{\log ^{3} d_{2}}{\sqrt{d_{1}}} \\
& \quad=\sum_{\substack{i+j+k=3 \\
i, j, k \geq 0}} a_{i j k}\left(\sum_{c \mid r_{1}} \Lambda_{i}(c) \tau\left(r_{1} / c\right)\right)\left(\sum_{c \mid r_{2}} \Lambda_{j}(c) \tau\left(r_{2} / c\right)\right)\left(\sum_{c \mid \alpha^{2} / e} \Lambda_{k}(c) \tau\left(\alpha^{2} / e c\right)\right) .
\end{aligned}
$$

Again the contribution of terms with $k \geq 1$ is $O\left(K \log ^{5} K\right)$. We have seen that $\sum_{c \leq x} \frac{\Lambda_{2}(c) \mu_{*}(c)}{c^{2} v_{c}} \ll \log x$. Similarly we have $\sum_{c \leq x} \frac{\Lambda_{3}(c) \mu_{*}(c)}{c^{2} v_{c}} \ll(\log x)^{2}$, by considering the cases in which $c$ is product of one, two or three distinct primes. Since every term of (4.46) with $k=0$ must have $i>1$ or $j>1$, we get that all of (4.45) is $O\left(K \log ^{5} K\right)$.

- The contribution of the $\log r_{1} r_{2} \log ^{2} d_{2}$ term of (4.27) to (4.26) is

$$
\frac{-\hat{h}(0) K}{2} \sum_{\alpha} \sum_{e \mid \alpha^{2}} \sum_{\substack{r_{1}, r_{2} \\\left(r_{1}, r_{2}\right)=1}} \frac{\sqrt{e}}{\alpha^{2} r_{1} r_{2}}\left(\sum_{d \mid r_{1}^{2} r_{2}^{2} \alpha^{4} / e^{2}} \frac{\log ^{2} d_{2}}{\sqrt{d_{1}}}\right) \log r_{1} x_{\alpha r_{1}} x_{\alpha r_{2}}
$$


By the same analysis used for the previous terms, this equals

$$
\begin{aligned}
& -\hat{h}(0) K \sum_{\alpha, \beta} \sum_{e \mid \alpha^{2}} \sum_{b, c} \frac{e^{\frac{1}{2}} \tau(\beta)^{2} \mu(\beta) \tau\left(\alpha^{2} / e\right)}{\alpha^{2} \beta^{2}} \frac{\Lambda(b)}{b} \frac{\Lambda(c)}{c} \\
& \quad \times \sum_{r_{1}, r_{2}} \frac{x_{\alpha \beta b r_{1}} x_{\alpha \beta c r_{2}} \tau\left(r_{1}\right) \tau\left(r_{2}\right) \log \left(b \beta r_{1}\right)}{r_{1} r_{2}}+O\left(K \log ^{5} K\right) \\
& \sim-\hat{h}(0) K \sum_{j} \sum_{b, c} v_{j} \frac{\Lambda(b)}{b} \frac{\Lambda(c)}{c}\left((\log b) y_{b j} y_{c j}+\sum_{d} \frac{\Lambda(d) \tau(d)}{d} y_{b d j} y_{c j}\right) .
\end{aligned}
$$

Using the definition of $y_{j}$ and partial summation, (4.48) is

$$
\begin{array}{r}
\sim-\hat{h}(0) K \sum_{j \leq M} \frac{\mu_{*}(j)^{2}}{j v_{j}^{2}}\left(\sum_{\substack{b \leq M / j \\
c \leq M / j}} \frac{\Lambda(b)^{2} \Lambda(c)}{b c} \frac{\mu_{*}(b) \mu_{*}(c)}{b v_{b} c v_{c}} \log (b K j) \log (c K j)\right. \\
\left.+\sum_{\substack{b d \leq M / j \\
c \leq M / j}} \frac{\Lambda(b) \Lambda(c) \Lambda(d) \tau(d)}{b c d} \frac{\mu_{*}(b d) \mu_{*}(c)}{b d v_{b d} c v_{c}} \log (b d K j) \log (c K j)\right) \\
\sim-\hat{h}(0) K \sum_{j \leq M} \frac{\mu_{*}(j)^{2}}{j v_{j}^{2}}\left(\left(\frac{1}{2} \log ^{2}(M / j) \log (K j)+\frac{1}{3} \log ^{3}(M / j)\right)(\log (M / j) \log (K j)\right. \\
\left.+\frac{1}{2} \log ^{2}(M / j)\right)-\left(\log ^{2}(M / j) \log (K j)+\frac{2}{3} \log ^{3}(M / j)\right)(\log (M / j) \log (K j) \\
\left.\left.+\frac{1}{2} \log ^{2}(M / j)\right)\right)
\end{array}
$$

$\sim \hat{h}(0) K \log ^{6} K \frac{a^{4}(3+2 a)^{2}}{72}$.

4.4. Remaining terms. We are left to show that (4.25) is small when $i_{0}+i_{3}+i_{4}<$ 3. Since $\log r_{1} r_{2}=\log r_{1}+\log r_{2}$ we have by symmetry that (4.25) is bounded up to a constant by

$$
\begin{aligned}
K \log ^{i_{0}} K \sum_{\alpha} \sum_{e \mid \alpha^{2}} \frac{\sqrt{e} \log ^{i_{1}}\left(\alpha^{2} / e\right)}{\alpha^{2}} \\
\sum_{d \mid r_{1}^{2} r_{2}^{2} \alpha^{4} / e^{2}} \frac{\log ^{i_{2}} d_{1} \log ^{i_{3}} d_{2}}{\sqrt{d_{1}}} \sum_{\substack{r_{1}, r_{2} \\
\left(r_{1}, r_{2}\right)=1}} \frac{x_{\alpha r_{1}} x_{\alpha r_{2}} \log ^{i_{4}} r_{1}}{r_{1} r_{2}} .
\end{aligned}
$$


Using (4.34) we see that this is bounded up to a constant by

$$
\begin{gathered}
\sum_{\substack{i_{2,1}+i_{2,2}+i_{2,3}=i_{2} \\
i_{3,1}+i_{3,2}+i_{3,3}=i_{3}}} K \log ^{i_{0}} K \sum_{\alpha} \sum_{e \mid \alpha^{2}} \frac{\sqrt{e} \log ^{i_{1}}\left(\alpha^{2} / e\right)}{\alpha^{2}} \sum_{d \mid \alpha^{4} / e^{2}} \frac{\log ^{i_{2,3}} d_{1} \log ^{i_{3,3}} d_{2}}{\sqrt{d_{1}}} \\
\sum_{\substack{a_{1}, b_{1}, a_{2}, b_{2}>0 \\
\Lambda_{i_{2,1}}\left(a_{1}\right) \Lambda_{i_{2,2}}\left(a_{2}\right) \Lambda_{i_{3,1}}\left(b_{1}\right) \Lambda_{i_{3,2}}\left(b_{2}\right)}}^{\left(a_{1} a_{2}\right)^{\frac{3}{2}} b_{1} b_{2}} \\
\sum_{\substack{r_{1}, r_{2} \\
\left(a_{1} b_{1} r_{1}, a_{2} b_{2} r_{2}\right)=1}} \frac{\tau\left(r_{1}\right) \tau\left(r_{2}\right) \log ^{i_{4}}\left(a_{1} b_{1} r_{1}\right)}{r_{1} r_{2}} x_{\alpha a_{1} b_{1} r_{1}} x_{\alpha a_{2} b_{2} r_{2} .} .
\end{gathered}
$$

Using the Mobius function to detect $\left(r_{1}, r_{2}\right)=1$ we get

$$
\begin{aligned}
& \sum_{\substack{r_{1}, r_{2} \\
\left(a_{1} b_{1} r_{1}, a_{2} b_{2} r_{2}\right)=1}} \frac{\tau\left(r_{1}\right) \tau\left(r_{2}\right) \log ^{i_{4}}\left(a_{1} b_{1} r_{1}\right)}{r_{1} r_{2}} x_{\alpha a_{1} b_{1} r_{1}} x_{\alpha a_{2} b_{2} r_{2}} \\
= & \sum_{\beta} \frac{\tau(\beta)^{2}}{\beta^{2}} \sum_{\substack{r_{1} \\
\left(a_{2} b_{2}, r_{1}\right)=1}} \frac{\log ^{i_{4}}\left(a_{1} b_{1} \beta r_{1}\right) \tau\left(r_{1}\right)}{r_{1}} x_{\alpha a_{1} b_{1} \beta r_{1}} \sum_{\substack{r_{2} \\
\left(a_{1} b_{1}, r_{2}\right)=1}} \frac{\tau\left(r_{2}\right)}{r_{2}} x_{\alpha a_{2} b_{2} \beta r_{2}} .
\end{aligned}
$$

Now we detect $\left(a_{2} b_{2}, r_{1}\right)=\left(a_{1} b_{1}, r_{2}\right)=1$ using the Mobius function again. Using the fact that $a_{1}, b_{1}, a_{2}$ and $b_{2}$ have at most three prime factors and the bound $y_{j} \ll \log K$, we have that (4.52) is bounded by a constant multiple of

$$
\begin{aligned}
& \sum_{\substack{c_{1}\left|a_{1} b_{1} \\
c_{2}\right| a_{2} b_{2}}} \sum_{\beta} \frac{\tau(\beta)^{2}}{\beta^{2}}\left(\log ^{i_{4}} K\left|y_{\alpha a_{1} b_{1} \beta c_{2}} y_{\alpha a_{2} b_{2} \beta c_{1}}\right|+\sum_{d} \frac{\Lambda_{i_{4}}(d)}{d}\left|y_{\alpha a_{1} b_{1} \beta c_{2} d} y_{\alpha a_{2} b_{2} \beta c_{1}}\right|\right) \\
& \ll \log ^{2+i_{4}} K .
\end{aligned}
$$

So (4.51) is

$$
\ll K \log ^{2+i_{0}+i_{3}+i_{4}} K \sum_{\alpha \leq M} \sum_{e \mid \alpha^{2}} \frac{\sqrt{e} \log ^{i_{1}}\left(\alpha^{2} / e\right)}{\alpha^{2}} \sum_{d \mid \alpha^{4} / e^{2}} \frac{\log ^{i_{2}} d_{1}}{\sqrt{d_{1}}} .
$$

The inner sum is

$$
\frac{1}{\alpha} \sum_{e \mid \alpha^{2}} \frac{\log ^{i_{1}} e}{\sqrt{e}} \sum_{d \mid e^{2}} \frac{\log ^{i_{2}} d_{1}}{\sqrt{d_{1}}} \ll \frac{1}{\alpha} \sum_{e \mid \alpha^{2}} e^{-\frac{1}{2}+\epsilon} .
$$

So (4.54) is $\ll K \log ^{3+i_{0}+i_{3}+i_{4}} K$ and we see that the terms with $i_{0}+i_{3}+i_{4}<3$ contribute only $O\left(K \log ^{5} K\right)$.

4.5. Completing the evaluation. We now complete the evaluation of $\mathcal{M}_{2}$ by adding together the contributions of each term of (4.27). The sum of (4.28), (4.32), 
(4.44), and (4.49) has the pleasing simplification:

$$
\begin{aligned}
& \hat{h}(0) K \log ^{6} K\left(\frac{a\left(3+3 a+a^{2}\right)}{18}+\frac{a^{2}(2+a)^{2}}{8}+\frac{a^{3}\left(6+7 a+2 a^{2}\right)}{12}+\frac{a^{4}(3+2 a)^{2}}{72}\right) \\
& (4.56) \\
& =\hat{h}(0) K \log ^{6} K \frac{a(1+a)^{3}\left(3+3 a+a^{2}\right)}{18} .
\end{aligned}
$$

4.6. Proportion of non-vanishing. Combining the evaluations of $\mathcal{M}_{1}$ and $\mathcal{M}_{2}$, we get for $a<\frac{1}{2}$ that

$$
\begin{aligned}
\frac{\mathcal{M}_{1}^{2}}{\mathcal{M}_{2}} & \sim \frac{\left(\hat{h}(0) K \log ^{3} K \frac{a\left(3+3 a+a^{2}\right)}{6}\right)^{2}}{\hat{h}(0) K \log ^{6} K \frac{a(1+a)^{3}\left(3+3 a+a^{2}\right)}{18}} \\
& =\left(1-\frac{1}{(1+a)^{3}}\right) \frac{\hat{h}(0) K}{2} \sim\left(1-\frac{1}{(1+a)^{3}}\right) \sum_{k \equiv 0 \bmod 2} h\left(\frac{k-1}{K}\right) \sum_{f \in H_{k}}^{h} 1 .
\end{aligned}
$$

Acknowledgements. I am grateful to Prof. K. Soundararajan for posing this problem and for many helpful discussions.

\section{REFERENCES}

1. Valentin Blomer, On the central value of symmetric square L-functions, Math. Z. 260 (2008), no. $4,755-777$.

2. J. B. Conrey and N. C. Snaith, Applications of the L-functions ratios conjectures, Proc. Lond. Math. Soc. (3) 94 (2007), no. 3, 594-646.

3. Freeman J. Dyson, Statistical theory of the energy levels of complex systems. I,II, and III, J. Mathematical Phys. 3 (1962), 140-175. MR MR0143556 (26 \#1111)

4. Stephen Gelbart and Hervé Jacquet, A relation between automorphic representations of GL(2) and GL(3), Ann. Sci. École Norm. Sup. (4) 11 (1978), no. 4, 471-542.

5. H. Iwaniec and P. Michel, The second moment of the symmetric square L-functions, Ann. Acad. Sci. Fenn. Math. 26 (2001), no. 2, 465-482.

6. Henryk Iwaniec, Topics in classical automorphic forms, Graduate Studies in Mathematics, vol. 17, American Mathematical Society, Providence, RI, 1997. MR MR1474964 (98e:11051)

7. Henryk Iwaniec, Wenzhi Luo, and Peter Sarnak, Low lying zeros of families of L-functions, Inst. Hautes Études Sci. Publ. Math. (2000), no. 91, 55-131 (2001).

8. Henryk Iwaniec and Peter Sarnak, The non-vanishing of central values of automorphic Lfunctions and Landau-Siegel zeros, Israel J. Math. 120 (2000), no. , part A, 155-177.

9. Nicholas M. Katz and Peter Sarnak, Random matrices, Frobenius eigenvalues, and monodromy, American Mathematical Society Colloquium Publications, vol. 45, American Mathematical Society, Providence, RI, 1999. MR MR1659828 (2000b:11070)

10. E. Kowalski and P. Michel, The analytic rank of $J_{0}(q)$ and zeros of automorphic L-functions, Duke Math. J. 100 (1999), no. 3, 503-542.

11. _ A lower bound for the rank of $J_{0}(q)$, Acta Arith. 94 (2000), no. 4, 303-343. MR MR1779946 (2003a:11054)

12. Yuk-Kam Lau, Non-vanishing of symmetric square L-functions, Proc. Amer. Math. Soc. 130 (2002), no. 11, 3133-3139 (electronic).

13. H. L. Montgomery, The pair correlation of zeros of the zeta function, Analytic number theory (Proc. Sympos. Pure Math., Vol. XXIV, St. Louis Univ., St. Louis, Mo., 1972), Amer. Math. Soc., Providence, R.I., 1973, pp. 181-193. MR MR0337821 (49 \#2590)

14. A. E. Özlük and C. Snyder, On the distribution of the nontrivial zeros of quadratic L-functions close to the real axis, Acta Arith. 91 (1999), no. 3, 209-228. MR MR1735673 (2001h:11116) 
15. Atle Selberg, Contributions to the theory of the Riemann zeta-function, Arch. Math. Naturvid. 48 (1946), no. 5, 89-155. MR MR0020594 (8,567e)

16. Goro Shimura, On the holomorphy of certain Dirichlet series, Proc. London Math. Soc. (3) 31 (1975), no. 1, 79-98.

17. K. Soundararajan, Nonvanishing of quadratic Dirichlet L-functions at $s=\frac{1}{2}$, Ann. of Math. (2) 152 (2000), no. 2, 447-488.

University of California at Los Angeles, Department of Mathematics, Los Angeles, CA 90095, USA

E-mail address: rrkhan@ucla.math.edu 